\title{
The origin of hydrogen line emission for five Herbig Ae/Be stars spatially resolved by VLTI/AMBER spectro-interferometry ${ }^{\star}$
}

\author{
S. Kraus ${ }^{1}$, K.-H. Hofmann ${ }^{1}$, M. Benisty ${ }^{2}$, J.-P. Berger ${ }^{2}$, O. Chesneau ${ }^{3}$, A. Isella ${ }^{4}$, F. Malbet ${ }^{2}$, A. Meilland ${ }^{1}$,

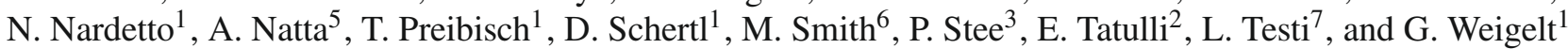 \\ 1 Max Planck Institut für Radioastronomie, Auf dem Hügel 69, 53121 Bonn, Germany \\ e-mail: skraus@mpifr-bonn.mpg.de \\ ${ }^{2}$ Laboratoire d'Astrophysique de Grenoble, UMR 5571 Université Joseph Fourier/CNRS, BP 53, 38041 Grenoble Cedex 9, France \\ UMR 6525 H. Fizeau, Univ. Nice Sophia Antipolis, CNRS, Observatoire de la Côte d'Azur, Av. Copernic, 06130 Grasse, France \\ Caltech, MC 105-24, 1200 East California Blvd., Pasadena CA 91125, USA \\ INAF - Osservatorio Astrofisico di Arcetri, Largo Fermi 5, 50125 Firenze, Italy \\ ${ }^{6}$ Centre for Astrophysics \& Planetary Science, University of Kent, Canterbury CT2 7NH, UK \\ 7 European Southern Observatory, Karl-Schwarzschild-Strasse 2, 85748 Garching, Germany
}

Received 10 April 2008 / Accepted 3 July 2008

\begin{abstract}
Context. Accretion and outflow processes are of fundamental importance for our understanding of the formation of stars and planetary systems. To trace these processes, diagnostic spectral lines such as the $\operatorname{Br} \gamma 2.166 \mu \mathrm{m}$ line are widely used, although due to a lack of spatial resolution, the origin of the line emission is still unclear.

Aims. Employing the AU-scale spatial resolution which can be achieved with infrared long-baseline interferometry, we aim to distinguish between theoretical models which associate the $\mathrm{Br} \gamma$ line emission with mass infall (magnetospheric accretion, gaseous inner disks) or mass outflow processes (stellar winds, X-winds, or disk winds).

Methods. Using the VLTI/AMBER instrument, we spatially and spectrally $(\lambda / \Delta \lambda=1500)$ resolved the inner $(\$ 5 \mathrm{AU})$ environment of five Herbig Ae/Be stars (HD 163296, HD 104237, HD 98922, MWC 297, V921 Sco) in the Br $\gamma$ emission line as well as in the adjacent continuum. From the measured wavelength-dependent visibilities, we derive the characteristic size of the continuum and Br $\gamma$ lineemitting region. Additional information is provided by the closure phase, which we could measure both in the continuum wavelength regime (for four objects) as well as in the spectrally resolved $\mathrm{Br} \gamma$ emission line (for one object). The spectro-interferometric data is supplemented by archival and new VLT/ISAAC spectroscopy.

Results. For all objects (except MWC 297), we measure an increase of visibility within the Bry emission line, indicating that the $\mathrm{Br} \gamma$-emitting region in these objects is more compact than the dust sublimation radius. For HD 98922, our quantitative analysis reveals that the line-emitting region is compact enough to be consistent with the magnetospheric accretion scenario. For HD 163296, HD 104237, MWC 297, and V921 Sco we identify an extended stellar wind or a disk wind as the most likely line-emitting mechanism. Since the stars in our sample cover a wide range of stellar parameters, we also search for general trends and find that the size of the $\mathrm{Br} \gamma$-emitting region does not seem to depend on the basic stellar parameters (such as the stellar luminosity), but correlates with spectroscopic properties, in particular with the $\mathrm{H} \alpha$ line profile shape.

Conclusions. By performing the first high-resolution spectro-interferometric survey on Herbig Ae/Be stars, we find evidence for at least two distinct $\mathrm{Br} \gamma$ line-formation mechanisms. Most significant, stars with a P-Cygni $\mathrm{H} \alpha$ line profile and a high mass-accretion rate seem to show particularly compact $\mathrm{Br} \gamma$-emitting regions $\left(R_{\mathrm{Br} \gamma} / R_{\mathrm{cont}}<0.2\right)$, while stars with a double-peaked or single-peaked $\mathrm{H} \alpha$-line profile show a significantly more extended $\mathrm{Br} \gamma$-emitting region $\left(0.6 \lesssim R_{\mathrm{Br} \gamma} / R_{\text {cont }} \lesssim 1.4\right)$, possibly tracing a stellar wind or a disk wind.
\end{abstract}

Key words. stars: pre-main sequence - stars: winds, outflows - planetary systems: protoplanetary disks - line: formation accretion, accretion disks - techniques: interferometric

\section{Introduction}

Accretion disks around young stellar objects (YSOs) are at the focus of astronomical research, not only because they play an essential role in the star-formation process, but also because they provide the stage where planet formation takes place.

Historically, these disks were discovered due to their characteristic infrared excess emission, which is believed to trace

* Based on observations made with ESO telescopes at the La Silla Paranal Observatory under open time programme IDs 077.C-0694, 078.C-0360, and 078.C-0680. dust grains, providing (for the expected temperatures and densities) the dominant source of opacity. In recent years, there has been substantial progress in constraining the detailed threedimensional geometry of the dust disk using, for example, combined modeling of the spectral energy distribution (SED) and spatially resolved infrared interferometric observations. While the thermal emission from the dust disk is likely to be the dominant contributor to the infrared excess emission observed towards YSOs, it is believed that the dust content makes up only a small fraction of the total mass of the system. $99 \%$ of the mass is likely contributed by gas, in particular hydrogen, and 
can mainly be traced by the spectral lines, which for pre-mainsequence stars are often found in emission. While some information about the kinematics of the gas can already be extracted from the line profile, the spatial origin of the gas emission and the physical processes they trace are still strongly debated.

In this context, the Brackett- $\gamma(\mathrm{Br} \gamma) 2.1661 \mu \mathrm{m}$ line is of special importance. It was found that the luminosity $L(\operatorname{Br} \gamma)$ of this line (as determined from the circumstellar component of the line equivalent width) seems to correlate with the mass accretion luminosity $L_{\text {acc }}$, as determined from UV veiling. This empirical $L(\mathrm{Br} \gamma)-L_{\text {acc }}$ relationship has been established for premain-sequence stars of various masses, including brown dwarfs (Natta et al. 2004), T Tauri stars (Muzerolle et al. 1998b), as well as intermediate-mass Herbig Ae/Be stars (Calvet et al. 2004; van den Ancker 2005). Based on this empirical correlation, observers started to use the $\operatorname{Br} \gamma$ luminosity as an estimator for the mass accretion rate (e.g. Garcia Lopez et al. 2006), which follows from $L_{\text {acc }}$ using $\dot{M}_{\text {acc }}=L_{\text {acc }} R_{\star} / G M_{\star}$.

Therefore, it is now essential to identify the process(es) involved in the formation of the $\mathrm{Br} \gamma$ emission line in YSOs. The main scenarios which have been proposed up to now include mass infall, as well as mass outflow mechanisms:

(a) Magnetospheric accretion: the line emission might emerge from matter which is accreted onto the star through magnetospheric accretion columns (van den Ancker 2005). This infall is supposed to happen very close to the star, inside the co-rotation radius, where the Keplerian angular velocity matches the stellar angular velocity.

(b) Gaseous inner disk: inside of the dust destruction radius, the gas continues to accrete towards the star, forming a gaseous inner accretion disk. The recombination line emission from ionized hydrogen in this disk might contribute to the line emission observed towards HAeBe stars. Muzerolle et al. (2004) estimated that especially for high mass-accretion rates $\dot{M}_{\text {acc }}>$ $10^{-7} M_{\odot} \mathrm{yr}^{-1}$, the flux contribution of the inner gaseous disk might be of importance.

(c) Stellar wind: the P Cygni line profile observed in the hydrogen lines of several HAeBe stars might also indicate mass loss through stellar winds (e.g. Mestel 1968; Catala \& Kunasz 1987; Strafella et al. 1998). While magnetically accelerated stellar winds seem plausible for massive stars rotating close to their break-up velocity, this scenario does not seem to work for lower-mass YSOs (Ferreira et al. 2006).

(d) Stellar-field driven wind (X-wind): in the $\mathrm{X}$-wind model (Shu et al. 1994), the outflows from YSOs are launched from a narrow interaction region where the stellar magnetosphere truncates the accretion disk. Keplerian rotation leads to a winding up of the field lines and to the formation of magnetic surfaces. Charged particles from the stellar wind will then get trapped in these surfaces, accelerated, and collimated into a beam.

(e) Disk-field driven wind (disk wind): another mechanism which has been proposed for the launching and collimation of outflows and jets observed towards YSOs are magnetocentrifugally driven disk winds (Blandford \& Payne 1982; Pudritz \& Norman 1983; Ferreira 1997). Material from the disk surface of the rotating disk is centrifugally accelerated along the open magnetic field lines of the accretion disk. In contrast to the Xwind model, where the wind is launched from a narrow disk annulus around the inner truncation radius of the accretion disk, disk winds originate from a wide range of radii, likely extending from the co-rotation radius out to several AUs.

Since most of these processes are believed to take place on (sub-)AU scales and were therefore not accessible with direct imaging techniques, most earlier studies tried to constrain the spatial distribution and kinematics of the line-emitting gas from the shape of line profiles. However, since these line profile fitting techniques are known to be highly ambiguous (e.g. Catala et al. 1999), spatially resolved observations are urgently required to constrain the physical mechanism and spatial origin of important tracer lines like $\operatorname{Br} \gamma$. Since the different emitting mechanisms noted above can be associated to distinct spatial regions (see Fig. 1), spatially resolved observations should allow us to identify and physically characterize the true underlying emitting mechanism.

The VLTI/AMBER instrument combines, for the first time, the milli-arcsecond spatial resolution achievable with IR longbaseline interferometry with a good spectroscopic resolution $(R=\lambda / \Delta \lambda=1500$ or 12000$)$ in the near-infrared $K$-band. In this study, we use the unique capabilities of this instrument in order to measure the geometry and position of the $\operatorname{Br} \gamma$ line-emitting region relative to the continuum-emitting region, providing direct information about the processes involved.

A particularly well-studied class of YSOs are the Herbig Ae/Be stars (HAeBes). These are intermediate-mass, pre-main sequence stars. Over the last few years, the continuum emission from a rather large number of HAeBes could already be studied with broad-band infrared interferometry. These studies revealed a correlation between the size of the NIR continuum emitting region and the stellar luminosity (Monnier \& Millan-Gabet 2002), suggesting that for most Herbig Ae stars, the NIR emission likely traces dust at the dust sublimation radius.

Only very little is known about the spatial origin of the hydrogen recombination line emission. First AMBER observations of the Herbig Be star MWC 297 have shown that the $\mathrm{Br} \gamma$-emitting region around this star is more extended than the continuum region (Malbet et al. 2007). Surprisingly, observations on the less luminous Herbig Ae star HD 104237 did not show any change in visibility along the $\operatorname{Br} \gamma$ line (Tatulli et al. 2007a). Eisner (2007) found a visibility increase within the Br $\gamma$ line for the Herbig Ae star MWC 480. This might suggest that the $\mathrm{Br} \gamma$ line traces fundamentally different mechanisms for Herbig Ae and Be stars. In this study, we present observations on three HAeBes, which are resolved for the first time with spectro-interferometry (HD 163296, HD 98922, V921 Sco). For HD 104237, we present new observations which were obtained at longer baseline lengths, adding substantial new information to the initial AMBER results presented by Tatulli et al. (2007a). Finally, we re-reduced the archival data on MWC 297 and HD 104237 in order to provide a uniformly calibrated sample of five stars for our interpretation. The stellar parameters which we adopted for the stars in our sample are shown in Table 1.

This paper is structured as follows: in Sect. 2 we discuss the spectro-interferometric and spectroscopic data and the applied data reduction procedures, followed by an outline of the obtained results (Sect. 3). Then, we present the models which were fitted to the data in order to constrain the geometry of the continuum(Sect. 4) and Br $\gamma$-emitting region (Sect. 5). After interpreting the individual objects in detail (Sect. 6), we discuss general trends 


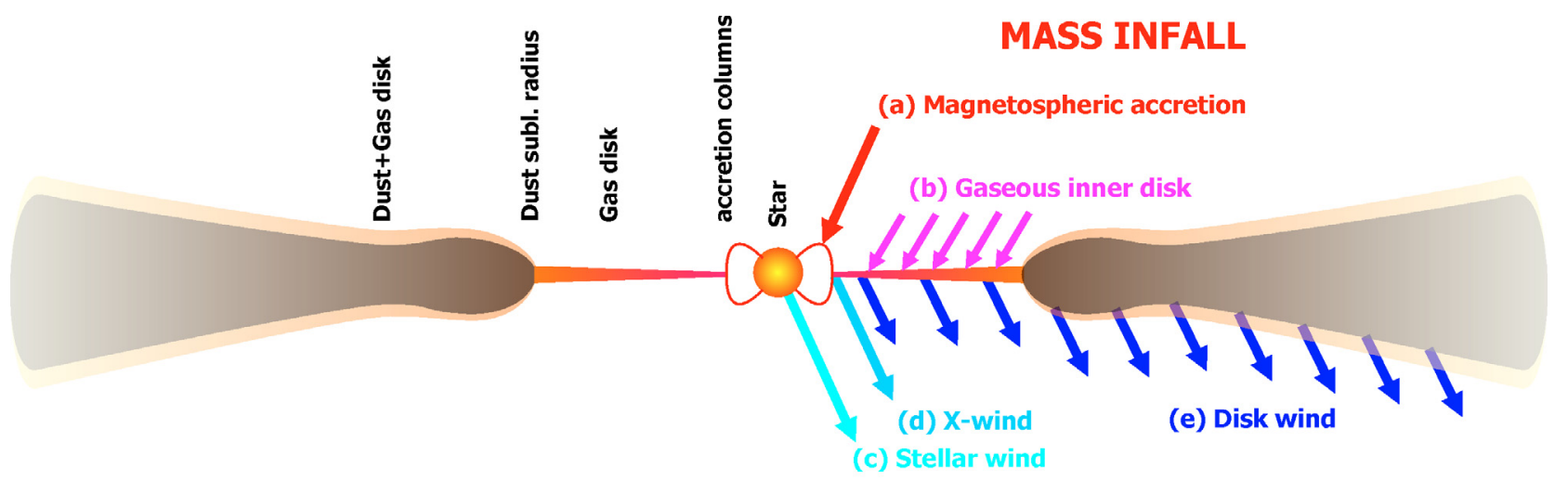

\section{MASS OUTFLOW}

Fig. 1. Illustration of the regions which have been proposed as the origin of the permitted hydrogen recombination line emission observed towards HAeBe stars (this sketch is not to scale; read Sect. 1 for details about the individual mechanisms).

Table 1. Target stars and adopted stellar parameters.

\begin{tabular}{|c|c|c|c|c|c|c|c|c|c|c|c|}
\hline$\overline{\text { Star }}$ & $\begin{array}{l}\text { Spectral } \\
\text { type }\end{array}$ & $\begin{array}{c}L_{\star} \\
{\left[L_{\odot}\right]}\end{array}$ & $\begin{array}{c}d \\
{[\mathrm{pc}]}\end{array}$ & $\begin{array}{l}T_{\star} \\
{[\mathrm{K}]}\end{array}$ & $A_{V}$ & $\begin{array}{c}R_{\star}{ }^{(a)} \\
{\left[R_{\odot}\right]}\end{array}$ & $\log \frac{L(\mathrm{~B} r \gamma)}{L_{\odot}}(b)$ & $\begin{array}{c}\log \dot{M}_{\mathrm{acc}}^{\mathrm{Br} \gamma(c)} \\
{\left[M_{\odot} \mathrm{yr}^{-1}\right]}\end{array}$ & $\begin{array}{c}v \sin i \\
{\left[\mathrm{~km} \mathrm{~s}^{-1}\right]}\end{array}$ & $\begin{array}{l}\mathrm{H} \alpha^{(d)} \\
\text { profile }\end{array}$ & Ref \\
\hline HD 104237 & A5 & 30 & $116^{(f)}$ & 8000 & 0.31 & 2.9 & -2.74 & -7.45 & $12 \pm 2^{(g)}$ & $\mathrm{D}^{(h)}$ & (i) \\
\hline HD 163296 & A3 & 26 & $122^{(f)}$ & 8700 & 0.12 & 2.2 & -2.78 & -7.12 & $120_{-30}^{+20}(j)$ & $\mathrm{D}^{(h)}$ & (k) \\
\hline HD 98922 & B9 & 890 & $540^{(e)}$ & 10600 & 0.3 & 9.1 & -1.56 & -5.76 & -30 & $\mathrm{P}^{(h)}$ & (l) \\
\hline MWC 297 & B1.5 & 10600 & 250 & 23700 & 8 & 6.1 & $>-0.6$ & - & $350 \pm 50^{(\mathrm{m})}$ & $\mathbf{S}^{(h)}$ & (m) \\
\hline V921 Sco & B0 & 19950 & 800 & 30900 & 5.0 & 5.0 & $>-0.7$ & - & - & $\mathrm{D}^{(n)}$ & (n) \\
\hline
\end{tabular}

Notes $-{ }^{(a)}$ The stellar radius $R_{\star}$ is computed using the given effective temperature and bolometric luminosity; ${ }^{(b)}$ luminosity of the Br $\gamma$ emission line, as determined by Garcia Lopez et al. (2006) from ISAAC spectra; ${ }^{(c)}$ mass accretion rate, as determined from the $L(\mathrm{Br} \gamma)-L_{\mathrm{acc}}$ relation (Garcia Lopez et al. 2006); ${ }^{(d)}$ this column described the $\mathrm{H} \alpha$-line profile shape of the stars in our sample using the classifications: D: doublepeaked profile; P: P-Cygni profile; S: single-peaked profile; ${ }^{(e)}$ for HD 98922, we assume the minimum distance given by Garcia Lopez et al. (2006).

References: $(f)$ van den Ancker et al. (1998); $(g)$ Donati et al. (1997); ( $h$ ) Acke et al. (2005); (i) Tatulli et al. (2007a); (j) Finkenzeller (1985); ( $k$ ) van den Ancker et al. (2000); (l) Garcia Lopez et al. (2006); (m) Drew et al. (1997); ( $n$ ) Habart et al. (2003).

which we find in our small sample of HAeBe stars (Sect. 7) in Sect. 8.

\section{Observations and data reduction}

\subsection{VLT/ISAAC spectroscopy}

The profile of the emission lines carries important information about the kinematics of the emitting gas. Therefore, we complement the spatially resolved AMBER spectro-interferometry with high-spectral resolution $(R \sim 9000)$ spectra obtained with the VLT/ISAAC instrument.

Besides archival ISAAC data (ESO programme 073.C-0184, P.I. Habart), we also obtained new spectroscopic data (for HD 163296 and V921 Sco, ESO programme 077.C0694, P.I. Kraus) in order to measure the line profile as close in time to the AMBER observations as possible. This new spectroscopic data covers not only the $\operatorname{Br} \gamma 2.1661 \mu \mathrm{m}$ line (Fig. 2, left), but also the $\mathrm{Pa} \beta 1.2822 \mu \mathrm{m}$ line (Fig. 2, right).

The raw spectra were extracted using IRAF procedures and then corrected for atmospheric features using telluric standard star observations obtained during the same night. For wavelength-calibration, we aligned the raw spectra to publicly available high-resolution $(R=40000)$ telluric spectra taken at the NSO/Kitt Peak Observatory. To compare the final ISAAC spectra (Fig. 2) with the spectra extracted from the AMBER data, we convolved the ISAAC spectra to the spectral resolution of AMBER $(R=1500)$ and found good agreement (see Figs. 3a to $7 \mathrm{a}$, top panel).

\subsection{Archival ISO and Spitzer/IRS spectroscopy}

In order to optimally constrain the SED for the stars in our sample, we obtained near- to mid-infrared spectroscopic data from the ISO and Spitzer Space Telescope archive. The Spitzer/IRS spectra were pre-processed by the S13.2.0 pipeline version at the Spitzer Science Center (SSC) and then extracted with the SMART software, Version 6.2.5 (Higdon et al. 2004).

\subsection{VLTI/AMBER spectro-interferometry}

AMBER (Petrov et al. 2007) is the NIR beam-combiner of the Very Large Telescope Interferometer (VLTI), which is located on Cerro Paranal/Chile and operated by the European Southern Observatory (ESO). Combining the light from up to three of the four $8.4 \mathrm{~m}$ unit telescopes simultaneously, AMBER measures not only visibility amplitudes, but also the closure phase $(\mathrm{CP})$ relation. In the course of three ESO open time programmes (077.C-0694, 078.C-0360, 078.C-0680, P.I. Kraus), we obtained spectrally dispersed interferograms in AMBER's medium resolution (MR) mode $(R=1500)$ on four HAeBes; namely, HD 163296, HD 104237, HD 98922, and V921 Sco. These new data sets were complemented with archival data from MWC 297 and HD 104237, obtained earlier during AMBER 
Table 2. Observation log of our VLTI/AMBER interferometric observations with a spectral resolution $R=1500$.

\begin{tabular}{|c|c|c|c|c|c|c|c|c|c|c|c|c|c|}
\hline \multirow[t]{2}{*}{ Target star } & \multirow{2}{*}{$\begin{array}{l}\text { Date } \\
\text { (UT) }\end{array}$} & \multirow{2}{*}{$\begin{array}{l}\text { Time } \\
\text { (UT) }\end{array}$} & \multirow{2}{*}{$\begin{array}{c}\text { Spectral } \\
\text { window } \\
{[\mu \mathrm{m}]}\end{array}$} & \multirow{2}{*}{$\begin{array}{l}\text { DIT } \\
{[\mathrm{ms}]}\end{array}$} & \multirow{2}{*}{$\begin{array}{c}\text { Telescope } \\
\text { triplet }\end{array}$} & \multicolumn{6}{|c|}{ Projected baselines } & \multirow{2}{*}{\multicolumn{2}{|c|}{ Calibrator $\quad$ Ref. }} \\
\hline & & & & & & $\begin{array}{c}B_{L} \\
{[\mathrm{~m}]}\end{array}$ & $\begin{array}{l}\mathrm{PA} \\
{\left[{ }^{\circ}\right]}\end{array}$ & $\begin{array}{c}B_{L} \\
{[\mathrm{~m}]}\end{array}$ & $\begin{array}{l}\mathrm{PA} \\
{\left[{ }^{\circ}\right]}\end{array}$ & $\begin{array}{c}B_{L} \\
{[\mathrm{~m}]}\end{array}$ & $\begin{array}{l}\mathrm{PA} \\
{\left[{ }^{\circ}\right]}\end{array}$ & & \\
\hline HD 104237 & $2005-02-26$ & $07: 14$ & $2.12-2.20$ & 100 & UT2-UT3-UT4 & 35.1 & 71 & 60.8 & 120) & $(87.9$ & $102)$ & HD 135382 & (a) \\
\hline HD 104237 & 2007-01-09 & 08:03 & $1.94-2.26$ & 200 & UT1-UT3-UT4 & $(78.0$ & 31) & 58.5 & 84 & (122.4 & $53)$ & HD 118934 & \\
\hline HD 104237 & 2007-01-09 & $07: 41$ & $1.94-2.26$ & 500 & UT1-UT3-UT4 & $(78.7$ & 26) & 58.0 & 78 & $(123.1$ & 47) & HD 118934 & \\
\hline HD 163296 & 2006-04-13 & $06: 19$ & $2.12-2.20$ & 50 & UT2-UT3-UT4 & 43.9 & 19 & 52.3 & 99 & 74.1 & 63 & $\begin{array}{l}\text { HD } 171960 \\
\text { HD } 89682\end{array}$ & \\
\hline HD 98922 & 2007-02-04 & 07:19 & $2.12-2.20$ & 50 & UT2-UT3-UT4 & 42.9 & 46 & $(61.9$ & 111) & $(88.9$ & $85)$ & HD 101328 & \\
\hline HD 98922 & 2007-01-09 & 09:31 & $1.94-2.26$ & 200 & UT1-UT3-UT4 & $(90.5$ & 42) & 62.3 & 116 & $(122.8$ & 71) & HD 118934 & \\
\hline HD 98922 & 2007-01-09 & 09:39 & $1.94-2.26$ & 500 & UT1-UT3-UT4 & (89.9 & 43) & 62.4 & 118 & $(122.0$ & 73) & HD 118934 & \\
\hline MWC 297 & 2004-05-31 & 06:02 & $2.00-2.23$ & 107 & UT2-UT3 & 44.7 & 42 & - & - & - & - & HD 177756 & (b) \\
\hline V921 Sco & 2006-04-14 & 08:09 & $2.12-2.20$ & 50 & UT2-UT3-UT4 & 45.2 & 42 & 62.1 & 110 & $(89.3$ & 82) & HD 159941 & \\
\hline
\end{tabular}

Notes - For more detailed information about the calibrator stars, we refer to Table 3.

References - (a) reprocessing of data presented in Tatulli et al. (2007a); (b) reprocessing of data presented in Malbet et al. (2007).

Table 3. Calibrator star information for the interferometric observations presented in Table 2.

\begin{tabular}{lcccc}
\hline \hline Star & $V$ & $K$ & $\begin{array}{c}\text { Spectral } \\
\text { type }\end{array}$ & $\begin{array}{c}d_{\mathrm{UD}} \\
{[\mathrm{mas}]}\end{array}$ \\
\hline HD 101328 & 7.44 & 3.75 & K4III & $1.00 \pm 0.01^{(a)}$ \\
HD 118934 & 7.92 & 4.02 & K4III & $0.89 \pm 0.01^{(a)}$ \\
HD 135382 & 2.88 & 2.53 & A1V & $1.10 \pm 0.08^{(b)}$ \\
HD 159941 & 7.85 & 3.55 & M0III & $1.09 \pm 0.02^{(a)}$ \\
HD 171960 & 7.29 & 3.38 & K3II & $1.13 \pm 0.02^{(a)}$ \\
HD 177756 & 3.43 & 3.56 & B9Vn & $0.60 \pm 0.06^{(b)}$ \\
\hline
\end{tabular}

Notes - The $V$-band magnitudes were taken from SIMBAD and the $K$-band magnitudes from the 2MASS point source catalog.

References - (a) UD diameter taken from the CHARM2 catalog (Richichi et al. 2005). (b) UD diameter computed with ASPRO.

commissioning and in guaranteed-time observations (Malbet et al. 2007; Tatulli et al. 2007a). Re-reducing these data sets with the latest software allows us to ensure homogenity both in data reduction as well as in the applied modeling procedures. The observations are summarized in Table 2 and were obtained under good atmospheric conditions (seeing 0.5-0.9", atmospheric coherence time $2-7 \mathrm{~ms}$ ). In order to calibrate the obtained visibilities and CPs for instrumental and atmospheric effects, we used interferometric calibrator stars, taking the intrinsic diameters of these stars into account (see Table 3). By using different detector integration times (DITs) for the different objects, we aimed for a compromise between collecting a sufficient number of photons and minimizing the loss of fringe contrast due to atmospheric piston. In general, observations with longer DIT should provide better SNR in the differential visibility measurement, while observations with shorter DIT should provide a better absolute calibration. Since we aimed mainly for a precise measurement of the differential quantities in the $\mathrm{Br} \gamma$ and the adjacent continuum, we used rather long DIT for most observations. In order to account for the resulting errors on the absolute calibration, we estimate a rather large calibration error of 5\%. The spectral calibration of the data was done by comparing the spectrum extracted from the AMBER data with ISAAC spectra of the same object (see Sect. 2.1), which have been convolved to the spectral resolution of AMBER. An additional very important calibration step in the course of AMBER data reduction was the relative spectral re-shifting of the photometric channels with respect to the interferometric channel. The appropriate shift was determined to sub-pixel accuracy by computing the auto-correlation between the spectra extracted from these channels.
In a first data reduction step, the AMBER raw interferograms were cleared from the correlated detector noise effect using the AMDC software tool (Version 1.1, Causi et al. 2008). Then, the raw data was reduced with the amdlib2 software $^{1}$ (release 2.1), employing the P2VM algorithm (Tatulli et al. 2007b). Due to the absence of a fringe tracker, a large fraction of the interferograms is of rather low contrast (see discussion in Petrov et al. 2007). Therefore, we removed any frames from our dataset for which the light injection from the contributing telescopes was unsatisfying; i.e., the intensity ratio between the photometric channels was larger than 4 , or the fringe contrast was decreased due to instrumental jitter (the $20 \%$ best interferograms were selected based on the Fringe SNR criteria, as defined in Tatulli et al. 2007b).

Since some stars in our sample are close to the current sensitivity limit of AMBER's MR mode, we applied spectral binning to the raw data in order to increase the fringe SNR. For each data set, the width of the sliding window was chosen so that the resulting fringe SNR of the interferograms exceeds the critical value of 1.5 , ensuring a reliable visibility estimation. Since the $\mathrm{Br} \gamma$ line for all our objects is spectrally resolved over several spectral channels at $R=1500$, the resulting decrease in spectral resolution only marginally reduces the contrast between the spectral line and the underlying continuum. This procedure increases the fringe SNR significantly, while the loss in spectral resolution (from $R=1500$ to 500 or 250) results only in a minor decrease of the line-to-continuum flux ratio $F_{\mathrm{Br} \gamma} / F_{\text {cont }}$. Baselines for which a spectral binning to $R=250$ was not sufficient to yield an SNR of 1.5 were rejected from further analysis (in Table 2, these baselines are put in brackets). The final visibility curves are shown in Figs. 3a to $7 \mathrm{a}$ in the second panel from the top.

In principle, two distinct phase quantities can be extracted from AMBER interferograms, namely differential phases (measuring the relative displacement of the line-emitting region with respect to the continuum-emitting region) and the closure phase, which can indicate asymmetries in the source brightness distribution. Since for most of our datasets, the fringe SNR in individual frames is not sufficient to reliably correct for disturbing atmospheric phase contributions (atmospheric piston), no scientifically meaningful differential phases could be extracted, unfortunately. However, for some data sets we could extract useful $\mathrm{CP}$ information, both in the continuum

\footnotetext{
1 The amdlib2 software package is available from the website http ://www.jmmc.fr/data_processing_amber.htm
} 


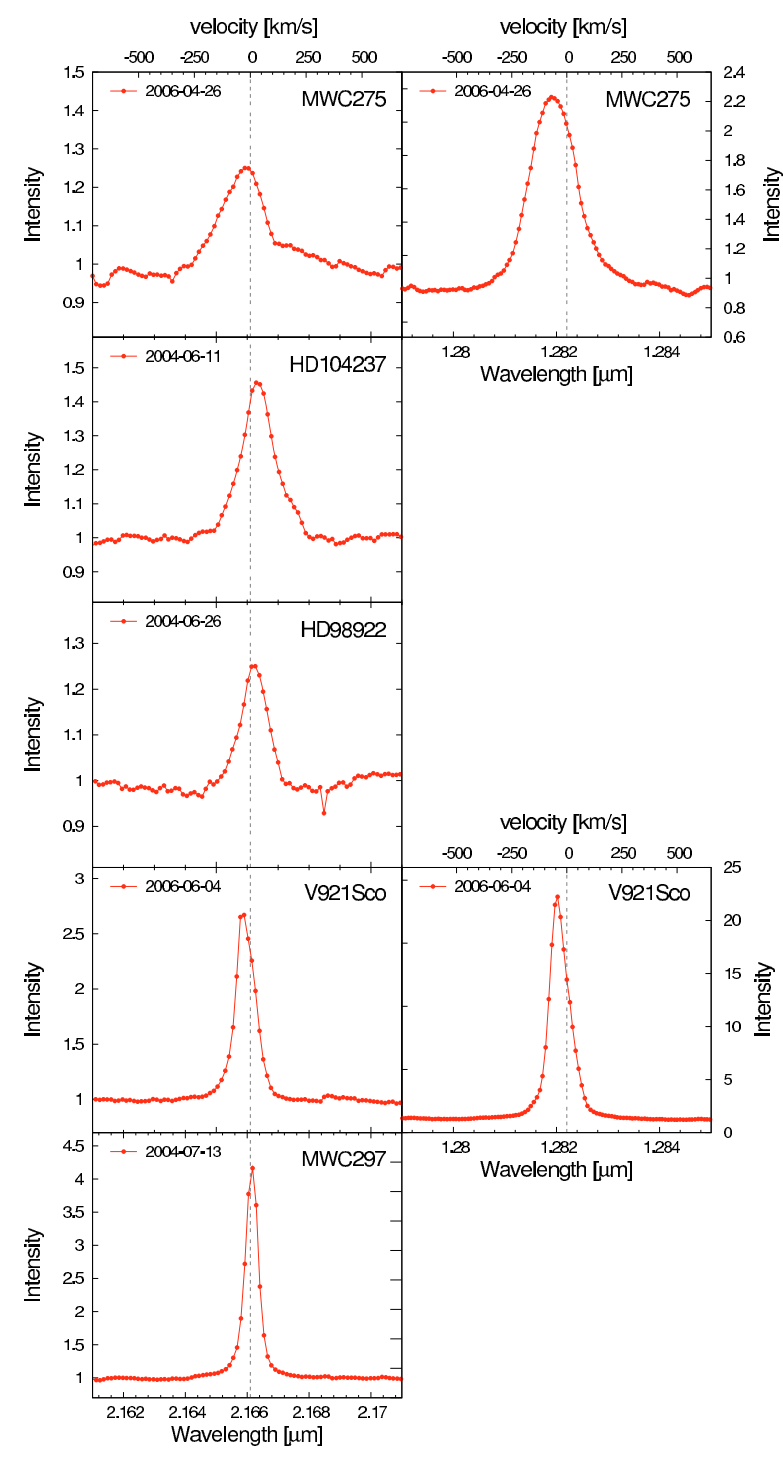

Fig. 2. Left: VLT/ISAAC spectra showing the $\mathrm{Br} \gamma$ line with a spectral resolution of $R \sim 9000$ for the stars in our sample. Right: VLT/ISAAC spectra of the $\mathrm{Pa} \beta$ line were obtained for HD 163296 and V921 Sco.

(HD 104237, HD 163296, HD 98922, V921 Sco) as well as in the line regime (V921 Sco). It is expected that the continuum $\mathrm{CP}$ does not change significantly over the small spectral window covered by our observations, which allowed us to apply an optimized data reduction strategy for the extraction of the continuum CPs. First, we apply some spectral binning to the raw data (bracketing out the spectral channels containing the emission line), followed by an averaging of the complex visibilities over various spectral channels (between 2.12-2.15 and $2.175-2.185 \mu \mathrm{m}$ ). The obtained CPs will be presented in Sect. 3.3.

\section{Results}

\subsection{SED and NIR-spectroscopy}

In order to constrain the SED of the stars in our sample, we collected photometric and spectroscopic data from the literature and the ISO and Spitzer archives (see Sect. 2.2). Assuming that the line-of-sight extinction can be mainly accounted to the large scale envelopes, in which some HAeBe stars are embedded, we
Table 4. Closure phases extracted from the data.

\begin{tabular}{l|cc|cc|c|c}
\hline \hline & \multicolumn{4}{|c|}{ Projected baselines } & \multicolumn{2}{c}{ Closure phase } \\
& $u_{12}$ & $v_{12}$ & $u_{23}$ & $v_{23}$ & continuum & Br $\gamma$ line \\
& {$[\mathrm{m}]$} & {$[\mathrm{m}]$} & {$[\mathrm{m}]$} & {$[\mathrm{m}]$} & {$[\mathrm{deg}]$} & {$[\mathrm{deg}]$} \\
\hline HD 104237 & 33.1 & 11.6 & 52.9 & -30.0 & $9.8 \pm 6.7$ & - \\
HD 163296 & 14.5 & 41.4 & 51.6 & -8.0 & $1.5 \pm 4.3$ & - \\
HD 98922 & 30.9 & 29.7 & 57.6 & -22.6 & $-9 \pm 16$ & - \\
V921 Sco & 30.2 & 33.7 & 58.3 & -21.6 & $20 \pm 24$ & $3.1 \pm 2.8$ \\
\hline
\end{tabular}

dereddened the photometric and spectroscopic data assuming the values for $A_{V}$ given in Table $1, R_{V}=3.1$, and the extinction law by Mathis (1990). The derived SEDs (Figs. 3b to 7b) allow us to determine the ratio between the star flux contribution $f_{\text {star }}$ and the flux contribution from the circumstellar disk $f_{\text {disk }}$ for the fitting of geometric models (Sect. 4).

The VLT/ISAAC Br $\gamma$ line spectra are shown in Fig. 2. With the currently available resolution, all $\mathrm{Br} \gamma$ lines show a singlepeaked profile. For HD 163296 and V921 Sco, measurements at two or three epochs are available, indicating that the line profile of V921 Sco has not significantly changed, while for HD 163296 we find some variability.

\subsection{Spectro-interferometric visibilities}

The wavelength-dependent visibilities extracted from our AMBER data are shown in the second panel from the top in Figs. 3a to 7a. Four stars in our sample (HD 163296, HD 104237, HD 98922, V921 Sco) show a visibility increase within the $\operatorname{Br} \gamma$ line. For MWC 297, the visibility drops within the line, as already reported by Malbet et al. (2007).

Since the spectral channels which include the $\operatorname{Br} \gamma$ line additionally contain flux contributions from the photosphere and circumstellar material, the interpretation of the measured wavelength-dependent visibilities requires quantitative modeling, as presented in Sect. 4.

\subsection{Closure phases}

Closure phase measurements can provide unique information about deviations from centro-symmetry in the source brightness distribution. For YSO disk geometries, such asymmetries are expected in particular for systems seen under intermediate inclination. The strongest $\mathrm{CP}$ signals are predicted by disk models with vertical puffed-up inner rim (e.g. Dullemond et al. 2001), while models with curved inner rims predict a smoother, more symmetric brightness distribution corresponding to smaller CPs (e.g. Isella \& Natta 2005).

Given the importance of this observable, we present here the CPs extracted from our data, although the error bars on most measurements are rather large, typically due to the low fringe contrast on the longest baseline in the telescope triplet. In Table 4 we list the CPs measured from our datasets and give the corresponding $(u, v)$ baseline vector for two baselines in the employed telescope triplet (the third $(u, v)$-vector is given by the closure relation). We find that all continuum CPs are consistent with a zero CP on the $1 \sigma$ (HD 163296, HD 98922, V921 Sco) or $2 \sigma$ level (HD 104237). This adds support to the conclusions drawn by Monnier et al. (2006), who used the IOTA-3T interferometer (providing baseline lengths up to $38 \mathrm{~m}$ ) and measured for 12 out of 13 sources CPs below $\sim 5^{\circ}$ (excluding one binary source). Since the CP signal is expected to increase rapidly with 
a) $\mathrm{V}(\lambda)$

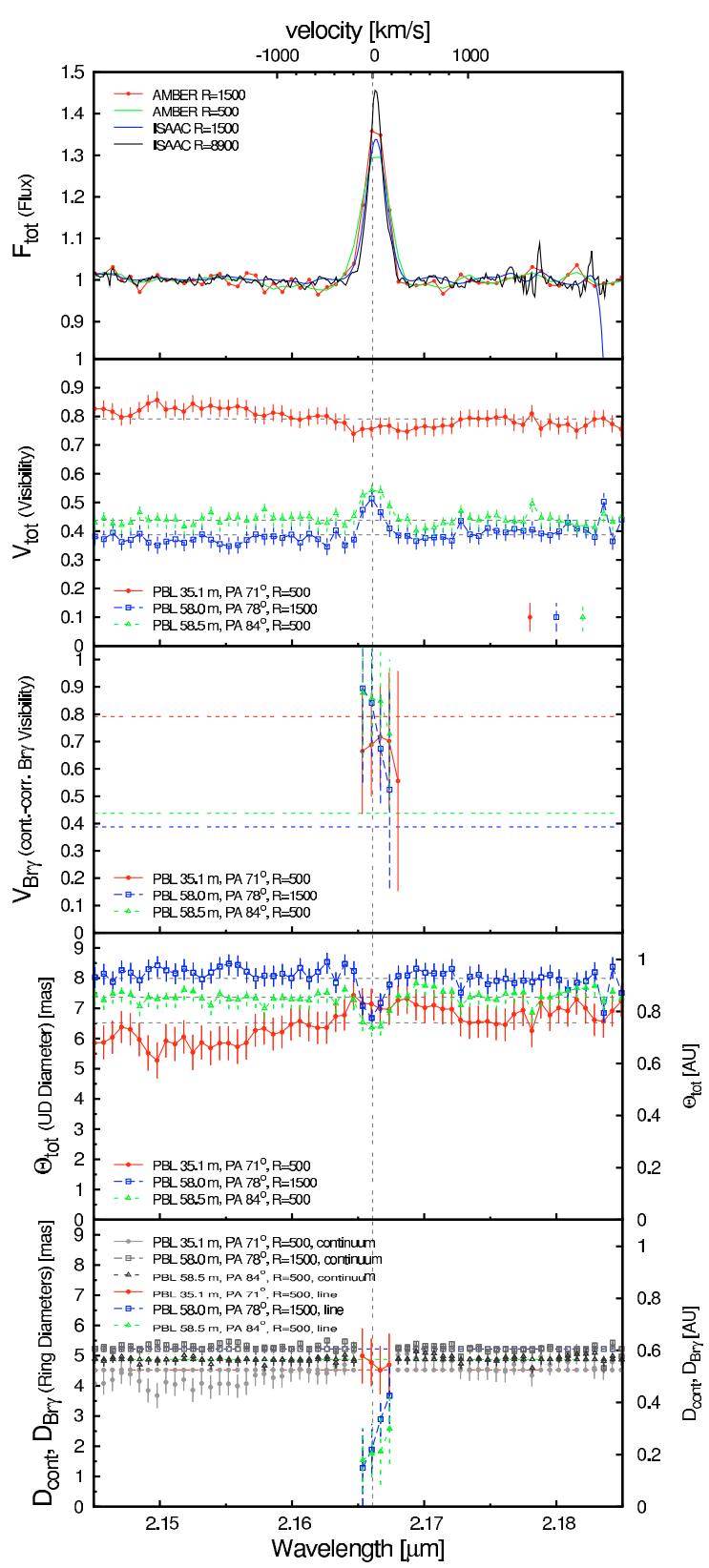

b) SED

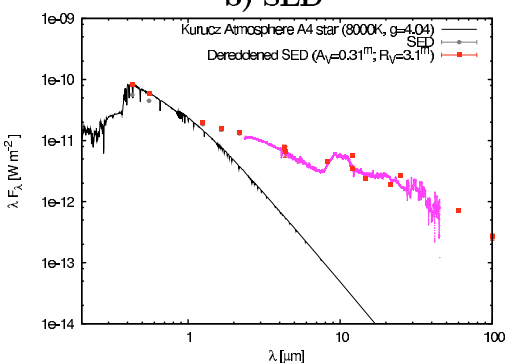

c) $\mathrm{V}_{\text {cont }}(\mathbf{B})$, continuum

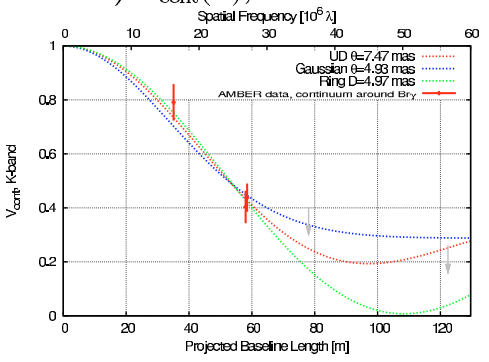

d) $\mathbf{V}_{\mathrm{Br} \gamma}(\mathbf{B})$, line

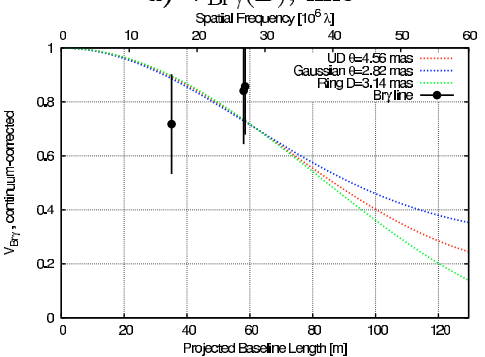

e) Geometric Models dRA [AU]

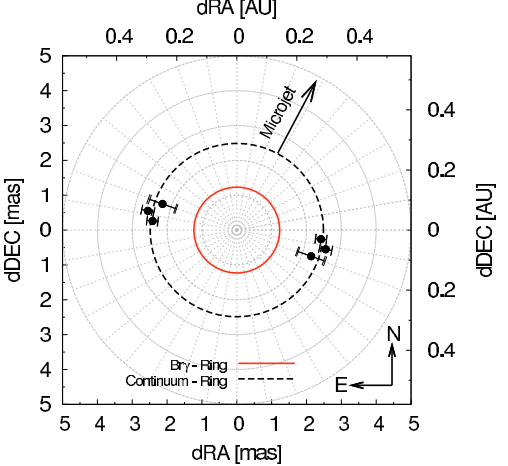

Fig. 3. Spectroscopic, spectro-interferometric, and photometric data for HD 104237: in a), we show the Br $\gamma$ spectra extracted from our ISAAC and AMBER data (top panel), the measured wavelength-dependent visibilities $V_{\text {tot }}$ for different baselines (2nd panel, the data points are shown with statistical errors bars, whereas the estimated calibration errors are shown in the bottom-right corner), the continuum-corrected Br $\gamma$-line visibility $V_{\mathrm{Br} \gamma}$ (3rd panel), the UD diameter $\Theta_{\text {tot }}$ derived from $V_{\text {tot }}$ (4th panel), and the diameters $D_{\text {cont }}$ and $D_{\mathrm{Br} \gamma}$ derived for the continuum- and the $\mathrm{Br} \gamma$-emitting region using a 2-ring model (5th panel). In b), the SED is shown, including photometric data from the literature (grey points), archival ISO spectra (magneta), and archival Spitzer/IRS spectra (blue). In c), we plot the measured $K$-band continuum-visibility as a function of baseline length and in d) the continuum-corrected $\mathrm{Br} \gamma$-line visibility. Panel e) shows the derived ring diameters for the continuum- and line-emitting region plotted as function of position angle.

baseline lengths, our new observations (with baselines up to $89 \mathrm{~m}$ ) provide new constraints on this issue and favour again a centro-symmetric brightness distribution. In Sect. 6, we discuss some of our CP measurements qualitatively, but leave it to future studies to investigate whether our results are also in quantitative agreement with the current generation of rim models.

For V921 Sco, the particularly strong Br $\gamma$ line flux allowed us also to measure an accurate $\mathrm{CP}$ measurement within the spectrally resolved emission line, yielding a CP signal of
$3.1 \pm 2.8^{\circ}$, consistent again with a centro-symmetric brightness distribution.

\section{Modeling: continuum-emission}

Over the last few years, ring models with uniform brightness have emerged as the prototypical geometry for the interpretation of YSO interferometric data. In most cases, this prefered use of ring geometries instead of other simple geometries 
a) $\mathbf{V}(\lambda)$

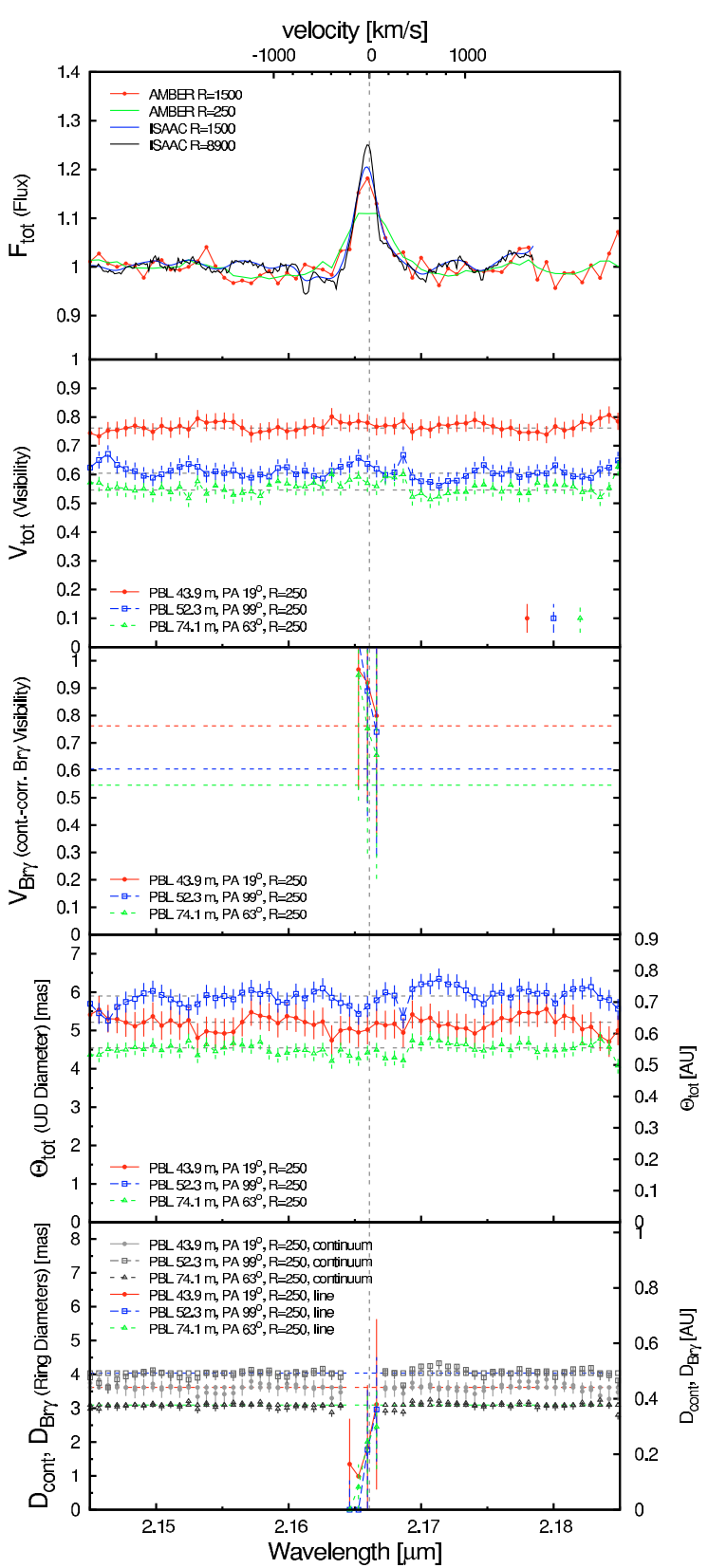

b) SED

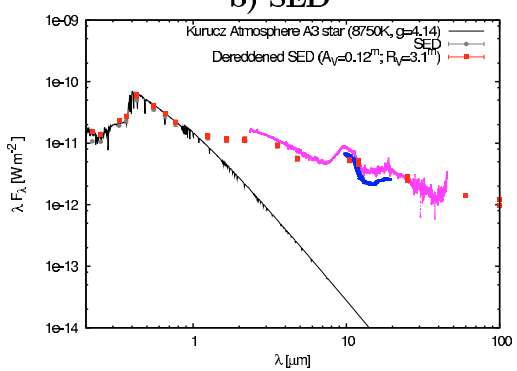

c) $\mathrm{V}_{\text {cont }}(\mathrm{B})$, continuum

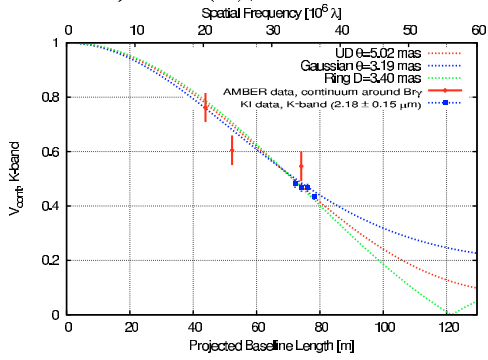

d) $\mathrm{V}_{\mathrm{Br} \gamma}(\mathrm{B})$, line

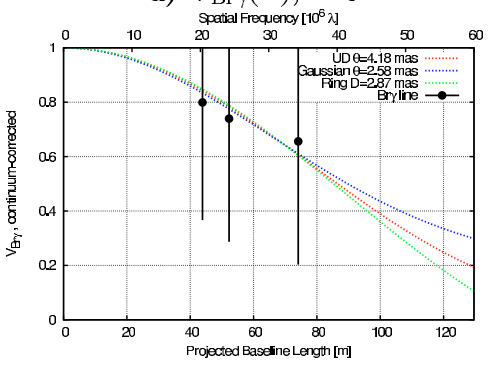

e) Geometric Models dRA [AU]

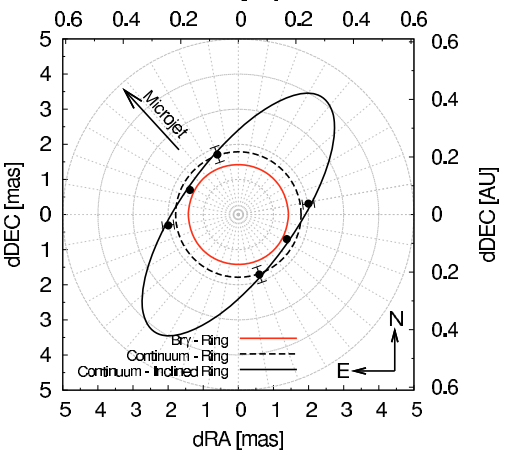

Fig. 4. Spectroscopic, spectro-interferometric, and photometric data for HD 163296 (similar to Fig. 3).

(e.g. uniform disk, Gaussian, or disk geometries with constant temperature power-law) is not explicitly required to reproduce the details of the sampled visibility function (which typically covers only the first lobe and is thus rather insensitive to the inner gap in the brightness distribution), but mainly based on indirect evidence or theoretical arguments. For example, some of the first infrared interferometric measurements on HAeBe stars (e.g. Millan-Gabet et al. 2001) already showed that classical geometrically thin accretion disk geometries extending down to several stellar radii might be consistent with interferometric measurements for early-type Herbig Be stars, but result in too compact structures for Herbig Ae and late-type Herbig Be stars (Eisner et al. 2004; Vinković \& Jurkić 2007). Ring geometries, on the other hand, yield sizes which are consistent with the expected dust sublimation radii and follow the predicted stellar luminosity scaling law (Monnier \& Millan-Gabet 2002). In the meantime, this finding was also interpreted in theoretical work (e.g. Natta et al. 2001; Dullemond et al. 2001; Isella \& Natta 2005), attributing the near-infrared continuum emission mainly to hot dust located at the dust sublimation radius. Based on these arguments, we also prefer to use ring-like geometries for the interpretation of our visibility data (assuming a fractional ring width of 20\%, Monnier et al. 2005), but also give uniform disk (UD) and Gaussian FWHM diameters to allow comparison with other work. In order to estimate the contribution of the stellar photosphere to the total $K$-band flux, we estimate the flux ratio using the SED and the Kurucz atmosphere models shown in Figs. $3 \mathrm{~b}$ to $7 \mathrm{~b}$. For more details about the model fitting procedure, we refer to one of our earlier studies (Kraus et al. 2008). 
a) $\mathbf{V}(\lambda)$

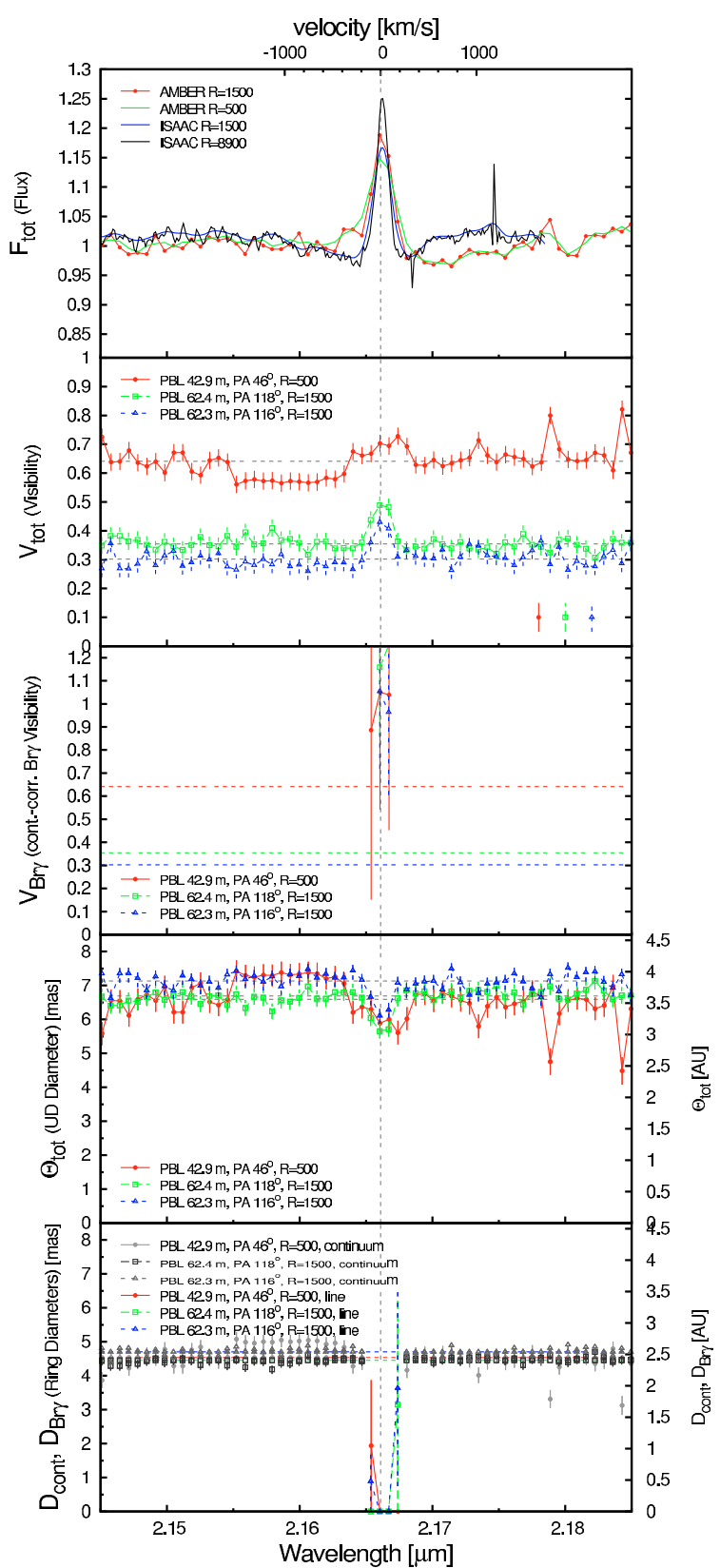

b) SED

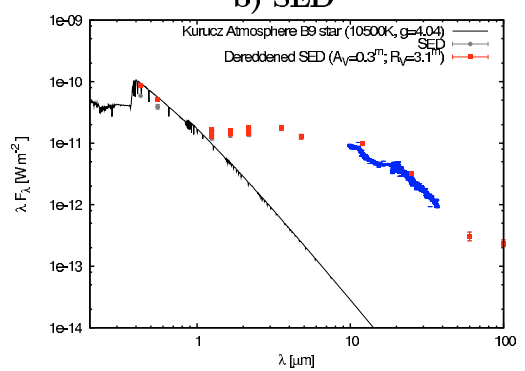

c) $\mathrm{V}_{\text {cont }}(\mathrm{B})$, continuum

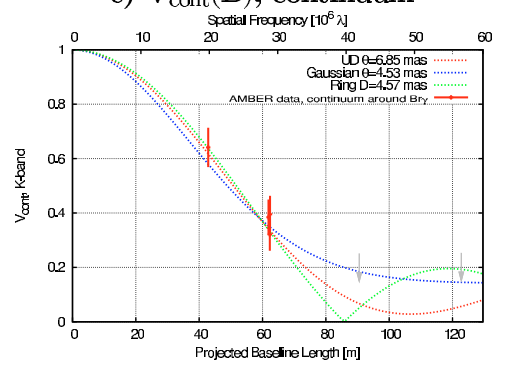

d) $\mathrm{V}_{\mathrm{Br} \gamma}(\mathrm{B})$, line

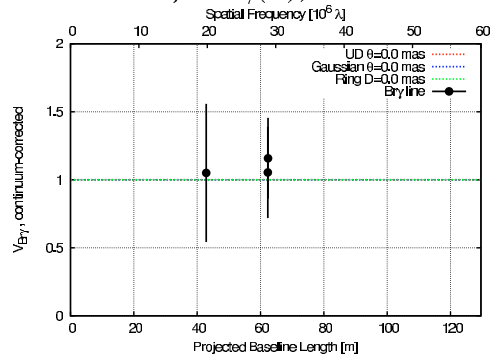

e) Geometric Models
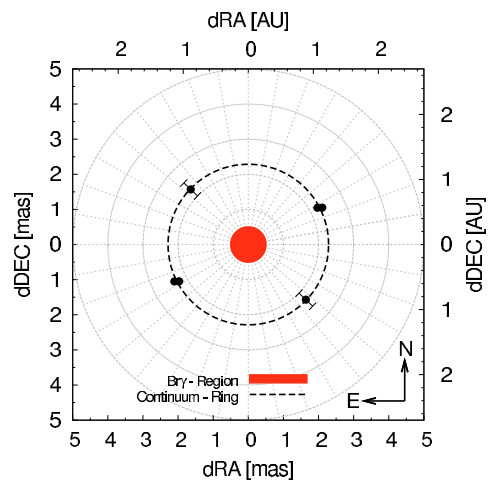

Fig. 5. Spectroscopic, spectro-interferometric, and photometric data for HD 98922 (similar to Fig. 3).

Besides model fits to each individual spectral channel (see fourth and fifth panel of Figs. 3a to 7a), we also fitted these models to continuum-visibilities, which were averaged over several spectral channels around 2.15 and $2.18 \mu \mathrm{m}$ and plot the corresponding measurements and model curves as a function of baseline length (Figs. 3c to $7 \mathrm{c}$ ). In these plots, we also show the upper limits, which we can put on the continuum visibility using the baselines which had to be rejected due to low SNR (see Sect. 2.3). The fitted diameters for Gaussian, UD, and ring profiles are listed in Table 5. To convert the measured angular size to physical scales, we assume the distances listed in Table 1. Please note that the errors given do not take distance uncertainties into account. By fitting the visibilities measured on different baseline orientations simultaneously, we assume that the intensity profile does not depend on position angle (e.g. face-on disk). Since the visibilities derived for HD 163296 provide sufficient position angle coverage to investigate for a possible elongation of the continuum-emitting region (e.g., due to disk inclination), we also fitted inclined ring geometries to this data set and derived an inclination of $68 \pm 10^{\circ}$ with a PA of $144 \pm 9^{\circ}$ (corresponding to an inclined ring diameter of $1.02 \times 0.37 \mathrm{AU})$.

\section{Modeling: $\mathrm{Br} \gamma$ line emission}

From each spectral channel of our AMBER interferograms, we can derive a value for the visibility amplitude, providing spatial information about the brightness distribution contributing to this spectral channel. Within the Br $\gamma$ line, the flux within a spectral channel is composed of the line emission plus the underlying continuum contribution. To model such visibility data, 
a) $\mathrm{V}(\lambda)$

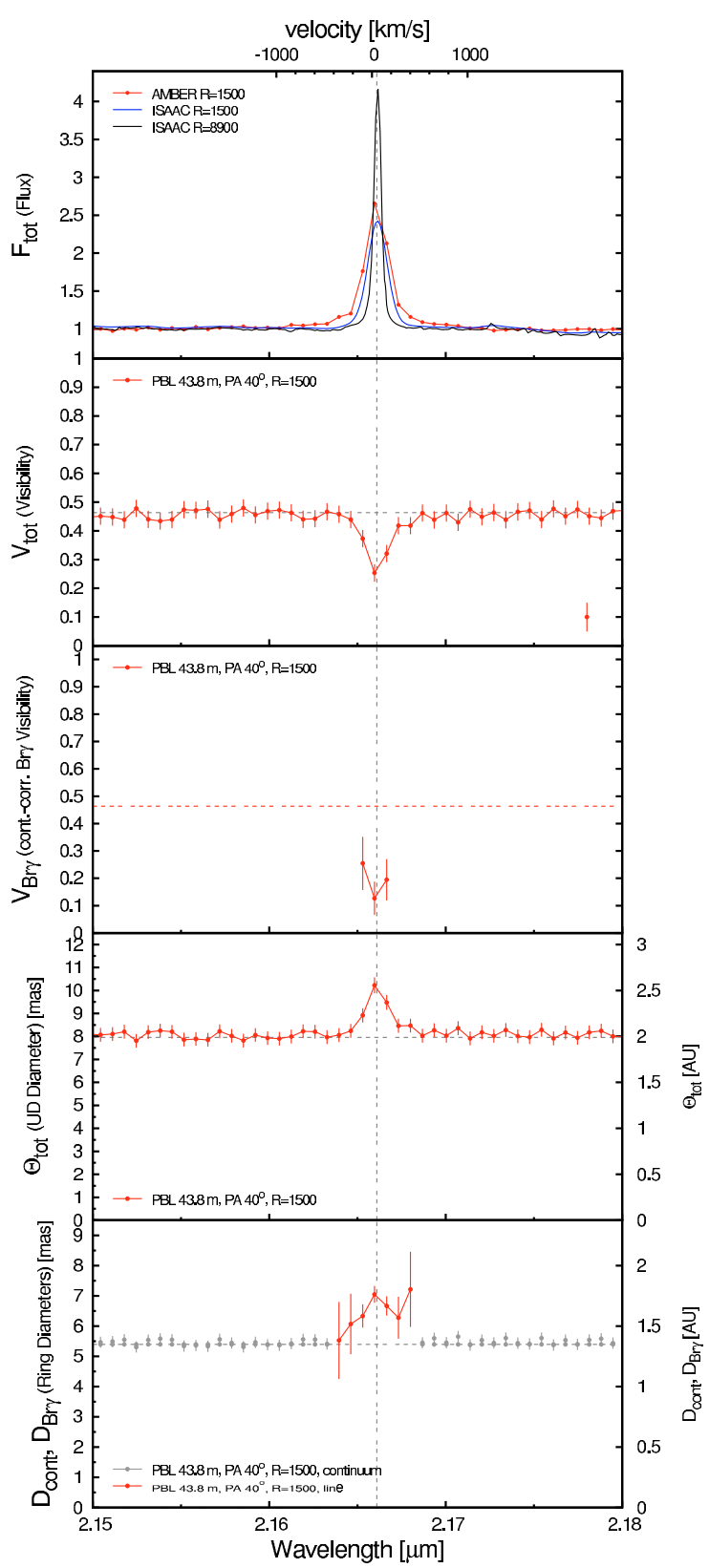

b) SED

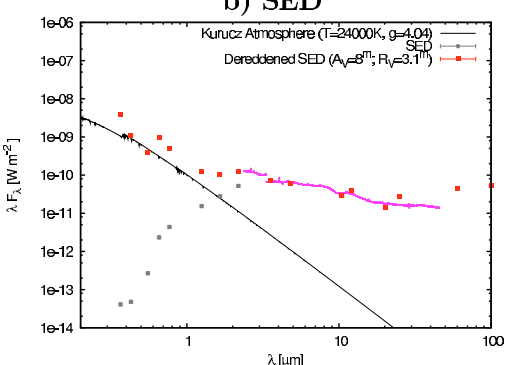

c) $\mathrm{V}_{\text {cont }}(B)$, continuum

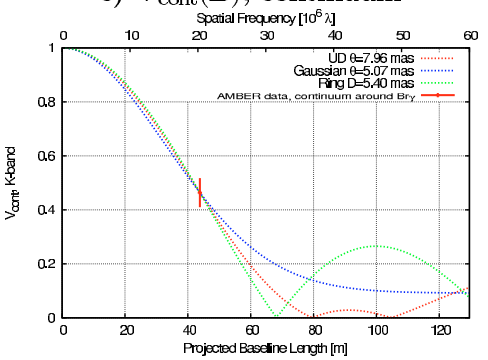

d) $\mathrm{V}_{\mathrm{Br} \gamma}(\mathrm{B})$, line

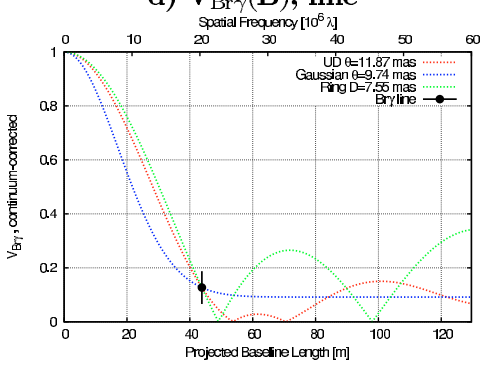

e) Geometric Models dRA [AU]

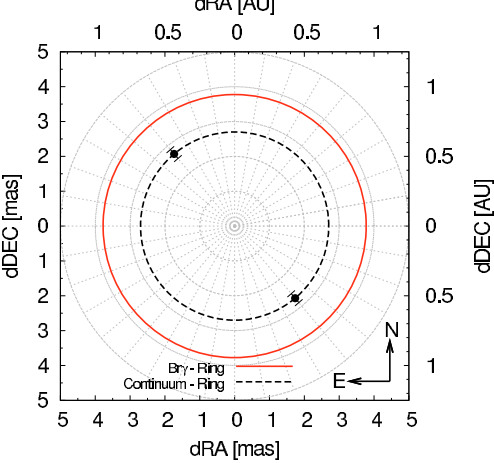

Fig. 6. Spectroscopic, spectro-interferometric, and photometric data for MWC 297 (similar to Fig. 3).

either the composite (line and continuum) object can be modeled, or the measured continuum+line visibility has to be corrected for the continuum contribution, yielding the visibility of the pure line-emitting region.

We correct the measured visibilities using the relation

$V_{\mathrm{Br} \gamma}=\frac{F_{\text {tot }} V_{\text {tot }}-F_{\text {cont }} V_{\text {cont }}}{F_{\mathrm{Br} \gamma}}$,

where $F_{\text {tot }}$ and $V_{\text {tot }}$ are the flux and the visibility measured within an arbitrary spectral channel. To compute the $\operatorname{Br} \gamma$-line flux $F_{\mathrm{Br} \gamma}:=F_{\text {tot }}-F_{\text {cont }}$, we determine the continuum flux $F_{\text {cont }}$ from Kurucz atmosphere models, taking the underlying photospheric $\mathrm{Br} \gamma$ absorption component into account. Then we interpolate the continuum visibility $V_{\text {cont }}$ over the $\mathrm{Br} \gamma$ line and derive the continuum-corrected visibility $V_{\mathrm{Br} \gamma}$ of the $\mathrm{Br} \gamma$-emitting region. As discussed in Weigelt et al. (2007, Appendix C), this relation assumes a negligible (zero) differential phase (corresponding to coinciding photocenters between the continuumand line-emitting regions). We compute the continuumcorrection for each spectral channel of the AMBER data where $F_{\mathrm{Br} \gamma}$ is sufficiently large to apply Eq. (1) reliably and show the resulting $V_{\mathrm{Br} \gamma}$ values in the third panel from the top of Figs. 3a to $7 \mathrm{a}$.

The interpretation of the derived line visibilities is difficult for several reasons. For instance, it is likely that the geometry of the line-emitting region is more complex than the continuum-emitting region, possibly extending above the equatorial disk plane, perhaps introducing strong inclination effects. Furthermore, as indicated by the short-period line variability detected towards YSOs of all masses, the kinematics and possibly also morphology of the line-emitting gas might change significantly even on short time scales of days or weeks, involving, 
a) $\mathbf{V}(\lambda)$

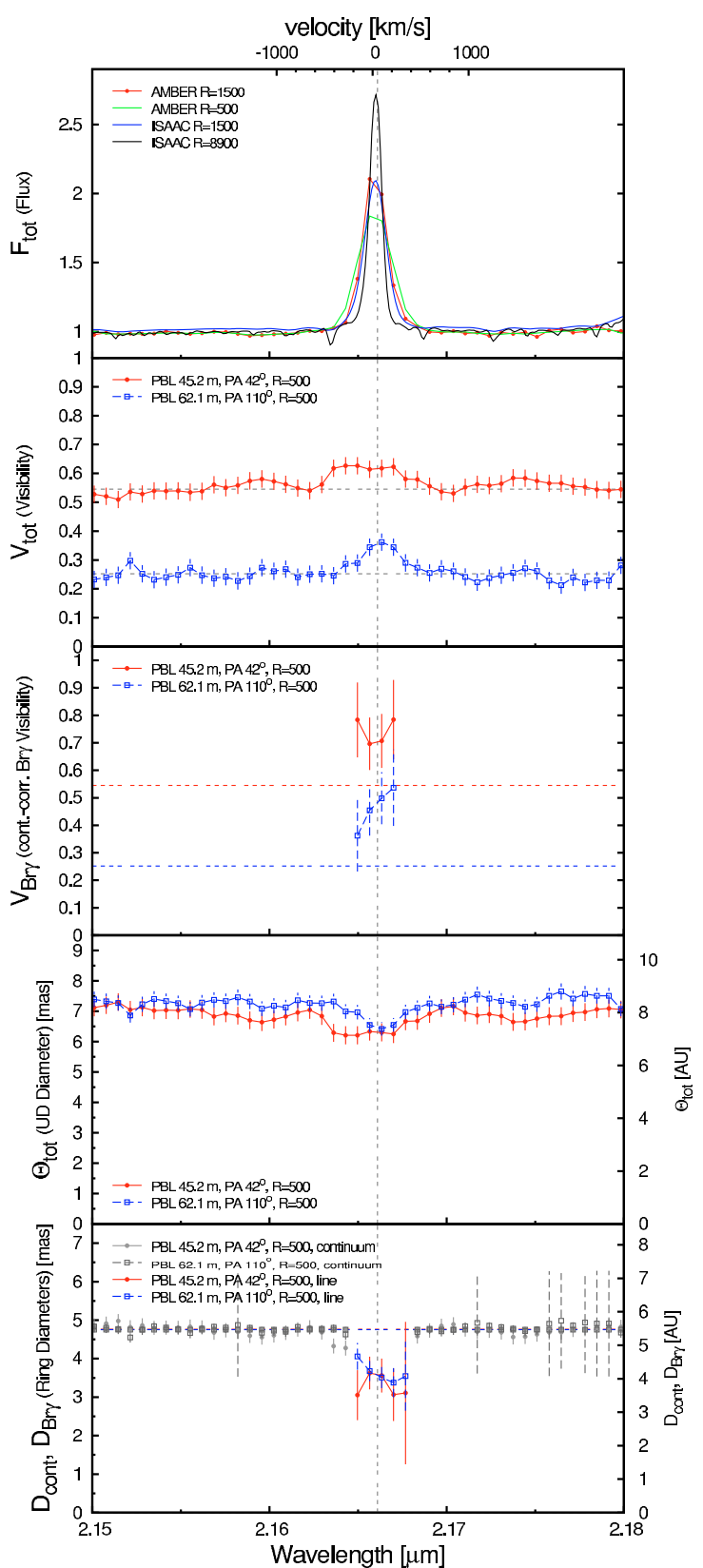

b) SED

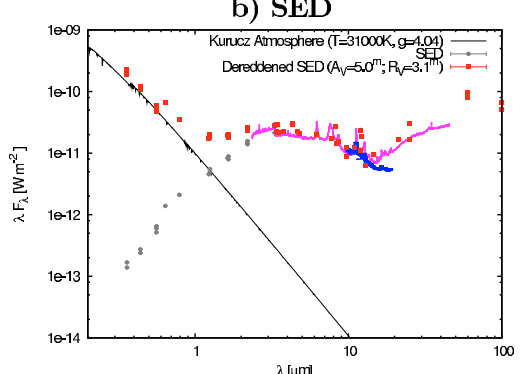

c) $\mathrm{V}_{\text {cont }}(\mathrm{B})$, continuum

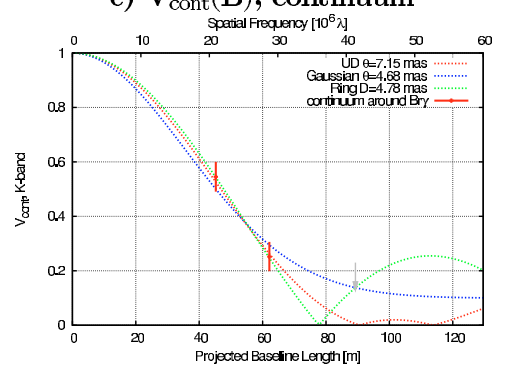

d) $\mathrm{V}_{\mathrm{Br} \gamma}(\mathrm{B})$, line

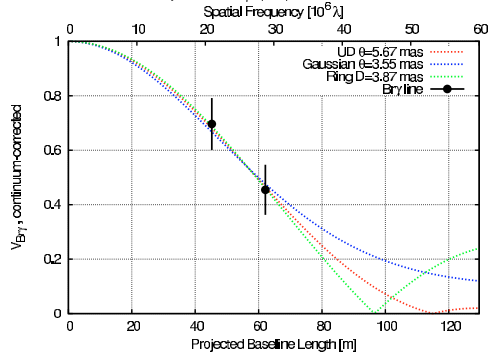

e) Geometric Models

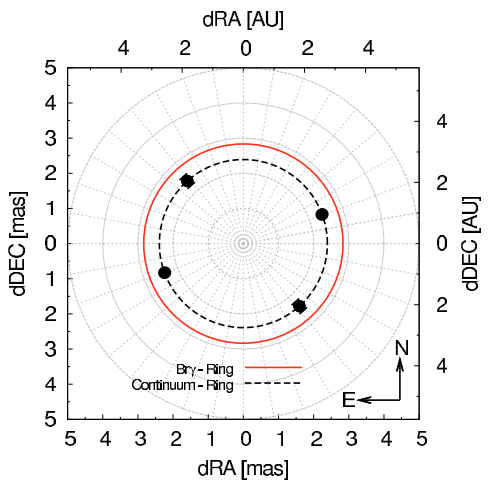

Fig. 7. Spectroscopic, spectro-interferometric, and photometric data for V921 Sco (similar to Fig. 3).

Table 5. Best-fit parameters for our geometric model fits.

\begin{tabular}{|c|c|c|c|c|c|c|c|c|c|c|}
\hline \multirow[b]{2}{*}{ target star } & \multicolumn{6}{|c|}{ Continuum emission } & \multicolumn{2}{|c|}{$\begin{array}{c}\text { Br } \gamma \text { emission } \\
\text { line center } \\
\text { ring }\end{array}$} & \multicolumn{2}{|c|}{$\begin{array}{c}\text { Br } \gamma \text { emission } \\
\text { all spectral channel } \\
\text { ring }\end{array}$} \\
\hline & $\begin{array}{c}F W H M_{\text {cont }} \\
{[\mathrm{AU}]}\end{array}$ & $\chi_{\text {red }}^{2}$ & $\begin{array}{l}\Theta_{\text {cont }} \\
{[\mathrm{AU}]}\end{array}$ & $\chi_{\text {red }}^{2}$ & $\begin{array}{l}D_{\text {cont }} \\
{[\mathrm{AU}]}\end{array}$ & $\chi_{\text {red }}^{2}$ & $\begin{array}{l}D_{\mathrm{Br} \gamma} \\
{[\mathrm{AU}]}\end{array}$ & $\chi_{\text {red }}^{2}$ & $\begin{array}{l}D_{\mathrm{Br} \gamma} \\
{[\mathrm{AU}]}\end{array}$ & $\chi_{\text {red }}^{2}$ \\
\hline HD 104237 & $0.57 \pm 0.10$ & 1.0 & $0.87 \pm 0.08$ & 0.5 & $0.58 \pm 0.04$ & 0.3 & $0.31 \pm 0.13$ & 0.6 & $0.35 \pm 0.21$ & 0.5 \\
\hline HD 163296 & $0.39 \pm 0.05$ & 0.9 & $0.61 \pm 0.08$ & 1.5 & $0.41 \pm 0.06$ & 1.9 & $0.35 \pm 0.15$ & 0.1 & $0.25 \pm 0.19$ & 0.1 \\
\hline HD 98922 & $2.45 \pm 0.26$ & 0.5 & $3.70 \pm 0.27$ & 0.2 & $2.47 \pm 0.16$ & 0.2 & $<0.5$ & - & $<0.5$ & - \\
\hline MWC 297 & $1.27 \pm 0.10$ & - & $1.99 \pm 0.13$ & - & $1.35 \pm 0.08$ & - & $1.89 \pm 0.52$ & 0.1 & $2.52 \pm 0.44$ & 0.4 \\
\hline V921 Sco & $5.38 \pm 0.52$ & 0.7 & $8.22 \pm 0.55$ & 0.1 & $5.49 \pm 0.32$ & 0.1 & $4.45 \pm 0.55$ & 0.1 & $4.35 \pm 0.64$ & 0.2 \\
\hline
\end{tabular}

Notes $-\chi_{\text {red }}^{2}$ is defined as $\sum\left[\left(V_{\text {meas }}-V_{\text {model }}\right) / \sigma_{V_{\text {meas }}}\right]^{2} /(N-1)$, where $N$ is the number of measurements, $V_{\text {model }}$ is the model visibility, and $V_{\text {meas }}$ and $\sigma_{V_{\text {meas }}}$ are the measured visibility and total error, respectively. 
for example, the ejection of fast-moving blobs at the base of an outflow. Finally, it is likely that several processes (including accretion and outflow processes) contribute to the $\operatorname{Br} \gamma$ emission. Given this complexity and the large error bars, we refrain from applying complex line radiative transfer models for the interpretation of the measured line visibilities in this first paper and instead limit ourselves to fitting simple ring-geometries, assuming one dominant source for the $\mathrm{Br} \gamma$ emission. Since the derived line visibility values are rather high for most objects, the characteristic size of the $\mathrm{Br} \gamma$-emitting region does not critically depend on the intensity profile of the underlying brightness distribution and therefore affects our conclusions only marginally. Since the $\mathrm{Br} \gamma$ line was spectrally resolved by AMBER in all observations, we performed two fits: one including all spectral channels where $V_{\mathrm{Br} \gamma}$ could be determined and one including only the spectral channel at line center. The circular-symmetric ring-model fits to the continuum-corrected $\mathrm{Br} \gamma$ visibilities are shown in Figs. $3 \mathrm{~d}$ to $7 \mathrm{~d}$, and the determined best-fit ring diameters are listed in Table 5.

\section{Discussion of individual objects}

\subsection{HD 104237: a circumbinary disk}

HD 104237 (alias DX Cha, CD-77528, Hen 3-741) has an approximate age of $2 \mathrm{Myr}$ (Böhm et al. 2004). Imaging observations by Grady et al. (2004) suggest that HD 104237 is associated with a microjet (HH 669) and that the disk is seen nearly face-on $\left(i=18_{-11}^{+14}\right.$ ). Spectroscopic monitoring suggests that HD 104237 consists of a binary system on a short-period (19.859 d), highly eccentric ( $e \approx 0.66$ ) orbit with component masses of $M_{A}=2.2 \pm 0.1 M_{\odot}$ and $M_{B}=1.7 \pm 0.1 M_{\odot}(\mathrm{Böhm}$ et al. 2004). Using these orbital elements, we estimate the major axis of the binary system to be $\sim 1.91$ mas $(0.23 \mathrm{AU})$, which places both stellar components inside of the ring-diameter measured by our AMBER observations at continuum wavelengths $(\sim 5.0$ mas $=0.58 \mathrm{AU})$. The measured continuum ring radius of $0.29 \mathrm{AU}$ is very close to the expected dust sublimation radius (0.32 AU, $L=30 L_{\odot}, T_{\text {subl }}=1500 \mathrm{~K}$, see Sect. 7.1), suggesting that the continuum emission traces mainly dust located at the inner rim of the circumbinary dust disk.

In the $\mathrm{Br} \gamma$ line, we measured no significant deviation of the line visibility with respect to the continuum visibility on 2005-02-26 at a short baseline length $\left(B_{p} \sim 35 \mathrm{~m}\right)$, while on 2007-01-09, the new measurements at $B_{p} \approx 58 \mathrm{~m}$ show a significant increase in visibility (see Fig. 3 ). In both epochs, the continuum-corrected $\mathrm{Br} \gamma$-line visibility indicates that the emitting region has a similar or smaller extension than the continuum-emitting region. In all available measurements, the $\mathrm{Br} \gamma$-emitting region is significantly more extended than the co-rotation radius, which leads us to reject the magnetospheric accretion scenario and favor the disk wind scenario (consistent with the conclusion from Tatulli et al. 2007a). Due to the low mass accretion rate of this object $\left(\log \dot{M}_{\text {acc }}=-7.45\right.$, Garcia Lopez et al. 2006), we deem major line contributions from a gaseous inner disk unlikely (Muzerolle et al. 2004).

For each epoch, the data cover only single baseline lengths, which makes it difficult to estimate the influence of the binary companion both on the continuum and the $\mathrm{Br} \gamma$ visibilities. Given our marginal detection of a non-zero continuum closure phase signal for HD 104237 (see Table 4) and the estimated binary parameters, it seems likely that we also detect small contributions from the inner resolved binary system. In particular, the binary nature might result in a significant asymmetry in the brightness distribution (in continuum and/or line emission) which could bias both the correction for the stellar contribution (see Sect. 3.1) as well as the continuum-correction required to compute $V_{\mathrm{Br} \gamma}$ (see Sect. 5 and Eq. (1)). Therefore, future investigations on this object will require a significantly larger amount of observational data to reliably discern the influence of these effects.

\section{2. $H D 163296$}

HD 163296 (alias MWC 275) is a particularly well-studied Herbig Ae star. CO millimeter line observations have revealed the outer parts of a Keplerian rotating disk (Mannings \& Sargent 1997; Isella et al. 2007). The determined disk inclination angle $\left(i=46 \pm 4^{\circ}\right)$ as well as the position angle $\left(\mathrm{PA}=128 \pm 4^{\circ}\right)$ are in reasonable agreement with the orientation of a disk-like structure seen in scattered light from coronographic imaging $(i=60 \pm$ $5^{\circ}, \mathrm{PA}=140 \pm 5^{\circ}$, Grady et al. 2000). Imaging observations have revealed an asymmetric, bipolar jet emerging roughly perpendicular to the disk plane and terminating in the Herbig-Haro objects $\mathrm{HH} 409$-A to $\mathrm{C}$, which were found along PA $=42.5 \pm$ $3.5^{\circ}$. The inclination angle inferred from the jet observations is $i=51_{-9}^{+11 \circ}$ (Wassell et al. 2006).

The infrared continuum environment around HD 163296 was already resolved with the VLTI/MIDI $(N$-band; $V(99 \mathrm{~m}) \approx$ 0.2 ; Leinert et al. 2004), IOTA ( $H$-band; $V(38 \mathrm{~m}) \approx 0.85$; Millan-Gabet et al. 2001), and the Keck interferometer ( $K$-band; $V(76 \mathrm{~m}) \approx 0.47$; Monnier et al. 2005). Recent measurements obtained with the CHARA interferometer (Tannirkulam et al. 2008) indicate that a significant fraction of the NIR continuum emission emerges from inside the dust sublimation radius, maybe due to gas contributions.

High-resolution spectroscopy $(R=25000)$ of the $\operatorname{Br} \gamma$ line of HD 163296 was presented by Brittain et al. (2007), showing a single-peaked line profile consistent with our ISAAC spectra. For the origin of the Balmer lines, Pogodin (1994) proposed a stellar wind/outer shell model.

In the $V(B)$-plot for the continuum wavelength regime (Fig. 4c), our AMBER measurements towards different baseline position angles (covering a PA range of $80^{\circ}$ ) cannot be well represented with the visibility profiles corresponding to ring-, UD-, or Gaussian-geometries, which might indicate either a very unusual radial intensity profile or, more likely, an elongation of the continuum-emitting region. The latter hypothesis can be investigated with the inclined ring model fits shown in Fig. 4e, indicating in fact that the near-infrared emitting region is strongly elongated. The major axis of the continuum-emitting region (PA $144 \pm 9^{\circ}$ ) seems to be oriented perpendicular to the outflow direction indicated by the optical microjet (PA $42.5 \pm$ $3.5^{\circ}$, Wassell et al. 2006). Due to the limited position angle coverage of our interferometric observations, the disk inclination angle $\left(i=68^{\circ}\right)$ is only poorly constrained by our observations, but seems to be in general in good agreement with the high inclination inferred from earlier observations. In spite of the strong inclination, the derived continuum closure phase for HD 163296 is still surprisingly close to zero (see Table 4), favouring disk models without notable inclination-induced skews.

As indicated by the quite flat wavelength-dependent visibility curve (Fig. 4a, second panel), the Br $\gamma$ emission originates in a region of similar spatial extent as the continuum-emitting region. On the longer baselines, we find weak evidence $(\sim 2 \sigma)$ for an increase of visibility within the $\mathrm{Br} \gamma$ line, which indicates that most of the line emission emerges from within the dust-sublimation radius. Plotting the continuum-corrected $\mathrm{Br} \gamma$-line visibility as a function of baseline length shows that the $\mathrm{Br} \gamma$-emitting region 
is likely spatially resolved (with $R_{\mathrm{Br} \gamma} / R_{\text {cont }} \approx 0.7$ ), which leads us to favor a stellar wind or a disk wind as the emitting mechanism. Considering the low mass accretion rate of HD 163296 $\left(\log \dot{M}_{\text {acc }}=-7.12\right.$, Garcia Lopez et al. 2006), it seems unlikely that the $\mathrm{Br} \gamma$ line emission emerges from a gaseous inner disk (Muzerolle et al. 2004).

\subsection{HD 98922}

For the B9Ve-type star HD 98922, a moderate infrared excess (Malfait et al. 1998) and a very high mass accretion rate was determined (Garcia Lopez et al. 2006; $\log \dot{M}_{\mathrm{acc}}=-5.76 M_{\odot} \mathrm{yr}^{-1}$, which is the highest mass accretion rate in the sample of 36 Herbig Ae stars investigated by these authors). Outflow signatures were detected in the $\mathrm{H} \alpha$ as well as in the SiII line $\left(v \approx 300 \mathrm{~km} \mathrm{~s}^{-1}\right.$, Grady et al. 1996). Acke et al. (2005) tried to model the profile of the [O I] emission line with a Keplerian rotating disk model and concluded that the emission likely does not originate in the disk surface as in several other sources, but possibly in a rotating gaseous disk inside the dust-sublimation radius. Van Boekel et al. (2003) studied the profile of the $10 \mu \mathrm{m}$ silicate emission feature detected towards HD 98922 and found spectral features associated with olivines and pyroxenes, indicating the presence of large, rather evolved grains.

The inclination under which HD 98922 is seen is still not well known. Blondel \& Djie (2006) modeled the UV spectrum and derived an intermediate inclination angle $\left(i \sim 45^{\circ}\right)$. Since the ring-diameters which we derive from our AMBER measurements are nearly identical towards very different position angles (Fig. 5a, lowest panel), we find no indications for a large disk inclination, but cannot exclude it either. Despite the fact that HD 98922 has the lowest observed $\mathrm{Br} \gamma$ line equivalent width of the stars in our sample $\left(F_{\mathrm{Br} \gamma} / F_{\text {cont }} \approx 1.2\right)$, we measure a strong increase of visibility within the $\mathrm{Br} \gamma$ line with respect to the continuum visibility (see Fig. 5). Remarkably, applying the continuum-correction to the measured $\mathrm{Br} \gamma$-line visibility reveals that the $\mathrm{Br} \gamma$ line-emitting region seems to be completely unresolved (i.e. $V_{\mathrm{Br} \gamma} \approx 1$ ) at baseline lengths up to $\sim 60 \mathrm{~m}$, while the continuum-emitting region is resolved with a ring-diameter of $4.6 \pm 0.1$ mas $(2.48 \pm 0.05 \mathrm{AU})$. Therefore, the Br $\gamma$-emitting region is at least a factor of 5 times more compact than the dustsublimation radius, which is consistent with the stellar wind, $\mathrm{X}$-wind, and magnetospheric accretion scenario. Given the spectroscopic indications for an exceptionally high mass accretion rate, we favor the magnetospheric accretion scenario.

\subsection{MWC 297}

MWC 297 (alias NZ Ser) is a B1.5Ve-type Herbig Be star with a remarkably high rotation velocity of $350 \pm 50 \mathrm{~km} \mathrm{~s}^{-1}$ (as derived from photospheric lines), suggesting that the system might be seen nearly edge-on (Drew et al. 1997). On the other hand, using spectro-polarimetry in the $\mathrm{H} \alpha$ line, Oudmaijer \& Drew (1999) could not find any evidence for asymmetry in the $\operatorname{Br} \gamma$ lineemitting region, as would be expected for a nearly edge-on system. Malbet et al. (2007) applied an analytic accretion disk model and could reproduce the SED and AMBER $K$-band and IOTA $H$-band visibilities with an inclination angle of $i \sim 20^{\circ}$ (i.e. closer to face-on than edge-on). On the other hand, Acke et al. (2008) investigated the near- and mid-infrared geometry of the circumstellar environment around MWC 297 and found best agreement using a two-component Gaussian geometric model.
The hydrogen line emission observed towards MWC 297 is exceptionally strong and has been interpreted as stellar wind (Nisini et al. 1995; Malbet et al. 2007) or accretion-driven massloss from the disk (Corcoran \& Ray 1998).

The UKIRT spectra of Drew et al. (1997) show a singlepeaked profile for the $\mathrm{H} \alpha, \mathrm{H} \beta$, and $\mathrm{Br} \alpha$ line. Garcia Lopez et al. (2006) presented an ISAAC spectrum of MWC 297, showing a double-peaked $\mathrm{Br} \gamma$-line profile. Based on our re-reduction of the ISAAC spectroscopic data, we cannot confirm the doublepeaked profile of the $\operatorname{Br} \gamma$ line, but classify the line profile as single-peaked with the currently available resolution $(R \sim$ 9000). NIR spectroscopy with higher spectral resolution will be required to determine the real underlying line profile.

As already discussed by various other authors (Monnier et al. 2005; Malbet et al. 2007), the continuum visibilities measured on MWC 297 indicate a very compact continuum-emitting region, corresponding to a ring-radius approximately 5-times more compact than the dust sublimation radius expected for an irradiated dust disk. The interpretation of this effect (which was also observed towards several other Herbig Be stars) is still debated, but might include either gas absorption (allowing dust to exist closer to the star; e.g. Monnier \& Millan-Gabet 2002; Malbet et al. 2007) or the emission of an optically thick gaseous inner accretion disk (e.g. Monnier et al. 2005; Kraus et al. 2008).

For MWC 297, the visibility drops significantly within the $\mathrm{Br} \gamma$ line (see Fig. 6), indicating that the line-emitting region is more extended than the continuum-emitting region. Malbet et al. (2007) modeled this observational result assuming an optically thick gas disk with an inner radius of $0.5 \mathrm{AU}$ and an outflowing stellar wind. Besides this interpretation, a disk wind scenario would likely also be consistent with our data. In this context, it is important to note that the pronounced drop of visibility within the $\mathrm{Br} \gamma$ line is also supported by the particular compactness of the continuum-emitting region of MWC 297. As discussed above, the continuum-emission around MWC 297 might not be dominated by pure dust emission, but instead by the emission of gas+dust located close to the star. Therefore, the observation that MWC 297 shows a drop of visibility within the $\mathrm{Br} \gamma$ line, while V921 Sco (which has a similar spectral type as MWC 297) shows an increase of visibility, might not indicate substantially different line formation mechanisms, but may simply reflect different physical conditions in the continuum-emitting dust and gas disk (e.g. optically thick vs. optically thin gas disk).

In case of a nearly edge-on system inclination, the influence of geometric effects (extension of the $\mathrm{Br} \gamma$-emission region above the mid-plane, appearance of bipolar lobes, etc.) would also be of importance for the interpretation and modeling of the spectro-interferometric data on MWC 297.

\subsection{V921Sco}

In the catalog of $\mathrm{HAeBe}$ member and candidate stars by de Winter \& The (1990), V921 Sco (alias MWC 865, CD$42^{\circ} 11721$, Hen $3-1300$ ) is classified as a B0[e]p-type star, although there is some dispute both about the stellar parameters and the evolutionary stage of this object (see discussion in Borges Fernandes et al. 2007). In the vicinity of V921 Sco, a cluster of YSO candidate sources were detected (Habart et al. 2003; Wang \& Looney 2007), and the star is also associated with an extended nebulosity, which was observed at optical (van den Bergh \& Herbst 1975; Hutsemekers \& van Drom 1990), near-infrared (Wang \& Looney 2007), mid-infrared (Natta et al. 1993), as well as sub-mm wavelengths (Henning et al. 1998). Comparing the spectral slope of the ISO spectrum 
with that of the Spitzer/IRS spectrum taken with a smaller aperture (see Fig. 7b) suggests that the SED at $\lambda \gtrsim 15 \mu \mathrm{m}$ is dominated by emission from this envelope. The SED model fits by Borges Fernandes et al. (2007) suggest that the system is seen under low to intermediate inclination $\left(i \lesssim 70^{\circ}\right)$.

The line profiles of the strong hydrogen recombination lines were modeled as emission from a spherically symmetric gas envelope (Benedettini et al. 1998). Acke et al. (2005) interpreted the profile of the optical [OI] line in the context of a wind originating from the surface layer of a passive disk.

Using the AMBER visibilities measured at continuum wavelengths, we compare the ring diameter measured towards various PAs (covering a position angle range of $\sim 68^{\circ}$ ) and find no indications for an elongation of the continuum-emitting region. This is consistent with the measured small $\mathrm{Br} \gamma$ line closure phase (see Table 4), indicating that the brightness distribution of the combined line- and continuum-emitting region is nearly centrosymmetric, as in the case when the line-emitting region is seen nearly face-on. Furthermore, V921 Sco exhibits a continuumemitting region which is more compact than expected for an irradiated dust disk, which might suggest that the NIR continuum emission is dominated by emission from an optically thick gaseous inner accretion disk (similar to MWC 297).

Within the $\mathrm{Br} \gamma$ line, we measure a slight increase in visibility (see Fig. 7). Furthermore, the continuum-corrected linevisibilities $V_{\mathrm{Br} \gamma}$ (ranging between 0.7 and 0.5 ) show that the $\mathrm{Br} \gamma$-emitting region is also spatially resolved and only slightly more compact than the continuum-emitting region $\left(R_{\mathrm{Br} \gamma} / R_{\text {cont }} \approx\right.$ 0.7). Therefore, the $\mathrm{Br} \gamma$ region is too extended to be consistent with magnetospheric accretion or an X-wind as dominant $\mathrm{Br} \gamma$-emitting mechanism, which makes a strong stellar wind, a disk wind or a gaseous inner disk the most likely scenario.

\section{Discussion of general trends}

Since our sample covers a wide range of stellar parameters, we investigated whether the measured size of the continuum and $\mathrm{Br} \gamma$-emitting region correlates with the stellar parameters or the spectroscopic properties. Revealing such relationships could provide more insight into the involved physical mechanisms and is also essential to confirm the empirically found correlations between the $\mathrm{Br} \gamma$ luminosity and other estimators for the mass accretion rate (Calvet et al. 2004). In order to further expand our sample, we include in this section not only the five objects which we have investigated with AMBER, but also the MWC 480 Keck-Interferometer measurement published by Eisner (2007). For MWC 480, we assume $L=17 L_{\odot}, d=$ $140 \mathrm{pc}, T_{\text {eff }}=8700 \mathrm{~K}$, a P-Cygni $\mathrm{H} \alpha$-line profile (Acke et al. $2005)$, and a $\operatorname{Br} \gamma$ line luminosity $\log \left(L(\operatorname{Br} \gamma) / L_{\odot}\right)=-2.8$ (as determined by Testi and Natta from unpublished TNG spectra; private communication).

\subsection{Continuum emission}

Earlier interferometric investigations of the continuum-emitting region of $\mathrm{HAeBe}$ stars have established a relation between the size of the continuum-emitting region and the stellar luminosity, suggesting that the continuum emission mainly traces hot dust located at the dust sublimation radius. In order to check for similar trends, we plot the determined $K$-band continuum ring radii as a function of the stellar luminosity $L_{\star}$ in Fig. 8. For comparison, we computed the predicted inner disk radii corresponding to dust sublimation temperatures $T_{\text {subl }}$ of $2000 \mathrm{~K}, 1500 \mathrm{~K}$, and

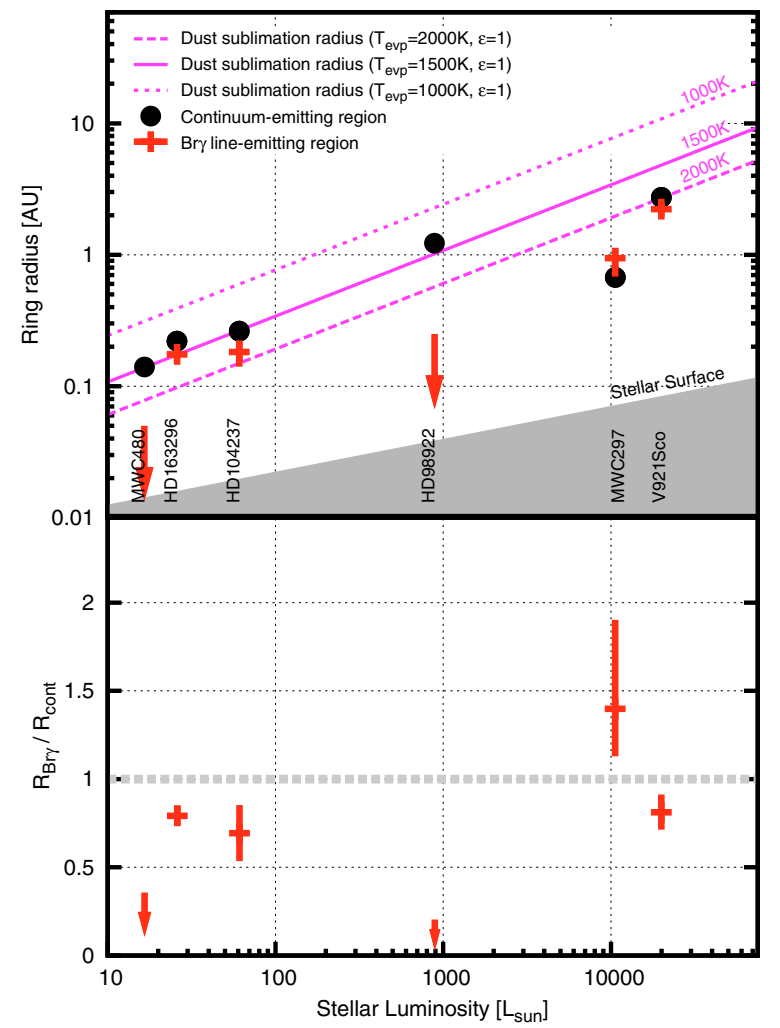

Fig. 8. Top: the fitted ring radii for the continuum ( $R_{\text {cont }}$, black points) and $\mathrm{Br} \gamma$ line ( $R_{\mathrm{Br} \gamma}$, red points) plotted as a function of stellar luminosity. For the $\mathrm{Br} \gamma$ line, we show the ring radius, determined by fitting only the spectral channel with the line center. For comparison, we plot the spatial extension of the stellar surface $R_{\star}$ (grey area) and the dust sublimation radius corresponding to dust sublimation temperatures of 2000, 1500, and $1000 \mathrm{~K}$ (computed using Eq. (2) and assuming $\epsilon=1$ ). Bottom: providing a natural measure for the temperature distribution within the circumstellar disk, we normalized $R_{\mathrm{Br} \gamma}$ by the size of the continuum-emitting region $R_{\text {cont }}$. In particular, this normalization seems important considering the large range of stellar luminosities covered by our sample.

$1000 \mathrm{~K}$ using the analytic expression for the dust sublimation radius $R_{\text {subl }}$ by Monnier \& Millan-Gabet (2002)

$R_{\text {subl }}=0.034 \mathrm{AU}\left(\frac{1500 \mathrm{~K}}{T_{\text {subl }}}\right)^{2} \sqrt{\frac{L_{\star}}{L_{\odot}} \frac{1}{\epsilon}}$.

In this relation, $\epsilon$ denotes the ratio of the absorption efficiencies of the dust at the sublimation temperature $T_{\text {subl }}$ and at the stellar effective temperature $T_{\star}$, i.e. $\epsilon=\kappa_{P}\left(T_{\text {subl }}\right) / \kappa_{P}\left(T_{\star}\right)$ with $\kappa_{P}(T)=$ $\int Q_{\mathrm{abs}}(\lambda) B_{\lambda}(T) \mathrm{d} \lambda / \int B_{\lambda}(T) \mathrm{d} \lambda$. We assume $\epsilon=1$, corresponding to rather large dust grains of several $\mu \mathrm{m}$ in size and, therefore, to the innermost region of the inner dust rim.

We find that the measured continuum radii of three of the five objects (HD 163296, HD 104237, HD 98922) follow the $R_{\text {subl }} \propto L_{\star}^{1 / 2}$ law rather closely assuming dust sublimation temperatures $T_{\text {eff }}$ between $1300 \mathrm{~K}$ and $1500 \mathrm{~K}$, which is in agreement with the results obtained in earlier NIR broadband interferometric survey observations (Monnier \& Millan-Gabet 2002). For the Herbig Be stars MWC 297 and V921 Sco, the derived ring diameters are significantly more compact than the expected dust sublimation radii. A possible explanation for the small apparent continuum sizes might be inclination effects, although, based on complementary information from the literature (see Sect. 6), we expect notable inclination only for HD 163296. 
Alternatively, the apparent disk size might be reduced either due to gas absorption (allowing dust to exist closer to the star) or due to the emission of optically thick gas located inside of the dustsublimation radius. As discussed in Sects. 6.4 and 6.5, we favor these latter explanations for MWC 297 and V921 Sco.

\subsection{Bry line emission}

\subsubsection{Correlations with stellar parameters}

Eisner (2007) speculated that the size of the Br $\gamma$-emitting region might depend on $L_{\star}$, with the (spatially compact) accretion processes dominating for stars with low luminosity and (spatially extended) outflow processes dominating in the highluminosity regime. Therefore, we investigate whether the derived physical size $\left(R_{\mathrm{Br} \gamma}\right)$ or the relative size of the line-emitting region $\left(R_{\mathrm{Br} \gamma} / R_{\mathrm{cont}}\right.$, i.e. normalized by the size of the continuumemitting region) scales with the stellar luminosity $L_{\star}$. As can be seen in Fig. 8, we do not find a clear correlation which includes all stars in our sample, although there might be a trend $R_{\mathrm{Br} \gamma} \propto$ $L_{\star}$, if we exclude the Herbig Be star HD 98922. Likewise, there might be a relation between $R_{\mathrm{Br} \gamma}$ and the stellar temperature $T_{\star}$ (Fig. 9, top), with a larger size of the Br $\gamma$-emitting region for higher effective stellar temperature. The fact that we have to exclude HD 98922 seems to indicate that $\mathrm{Br} \gamma$ traces not one unique physical mechanism for all stars in our sample, but at least two line-emitting mechanisms. As we argue in Sect. 6 and in the following subchapters, these mechanisms might include accretion and outflow processes, where the latter seem to be responsible for the tentative correlations with the stellar parameters noted above. However, given the small statistical sample, more observations will be required to confirm these first trends.

\subsubsection{Correlations with spectroscopic parameters}

Concerning the spectroscopic parameters, we search for correlations between $R_{\mathrm{Br} \gamma}$ and $R_{\mathrm{Br} \gamma} / R_{\text {cont }}$ and the $\mathrm{Br} \gamma$ line luminosity $L(\mathrm{Br} \gamma)$. Garcia Lopez et al. (2006) determined this quantity for all stars in our sample from the Bry line equivalent width of the circumstellar component. In principle, the accretion luminosity and mass accretion rate can be determined from $L(\mathrm{Br} \gamma)$ using the relation by Calvet et al. (2004) $\left(\log L_{\text {acc }} / L_{\odot}=0.9 \times\right.$ $\left.\left.\left(\log \left(L_{\mathrm{Br} \gamma} / L_{\odot}\right)+4\right)-0.7\right)\right)$. However, since applying this relation for the B-type stars in our sample would require to extrapolate the empirical $L(\mathrm{Br} \gamma)-L_{\text {acc }}$ relation well outside the range of stellar properties (luminosity and mass) of the calibration sample, we refrain from this step and use the line luminosity instead. There seems to be a general trend, that with increasing line luminosity the size of the $\mathrm{Br} \gamma$-emitting region increases (Fig. 10). In 2001, Muzerolle et al. already proposed a correlation between $W(\mathrm{Br} \gamma)$ (and thus also $L(\mathrm{Br} \gamma)$ ) and the size of the $\mathrm{Br} \gamma$-emitting region in the context of their magnetospheric accretion model for T Tauri stars (the low-mass counterparts of HAeBe stars). In order to explain the empirical $W(\mathrm{Br} \gamma)-L_{\text {acc }}$ correlation, they propose non-axisymmetric accretion, where the emitting area of discrete funnel flows increases with increasing accretion rate. However, since these funnel flows should still be located within a few stellar radii, we interpret the measurement of rather extended $\mathrm{Br} \gamma$-emitting regions in four out of six HAeBe stars as evidence against the hypothesis that $\mathrm{Br} \gamma$ is predominantly tracing magnetospheric accretion in these stars. It is also interesting to note that the width of the $\mathrm{Br} \gamma$ line seems to decrease with increasing stellar temperature (see Fig. 2). Stee \& Bittar (2001) have shown that this could be explained by optical depth effects

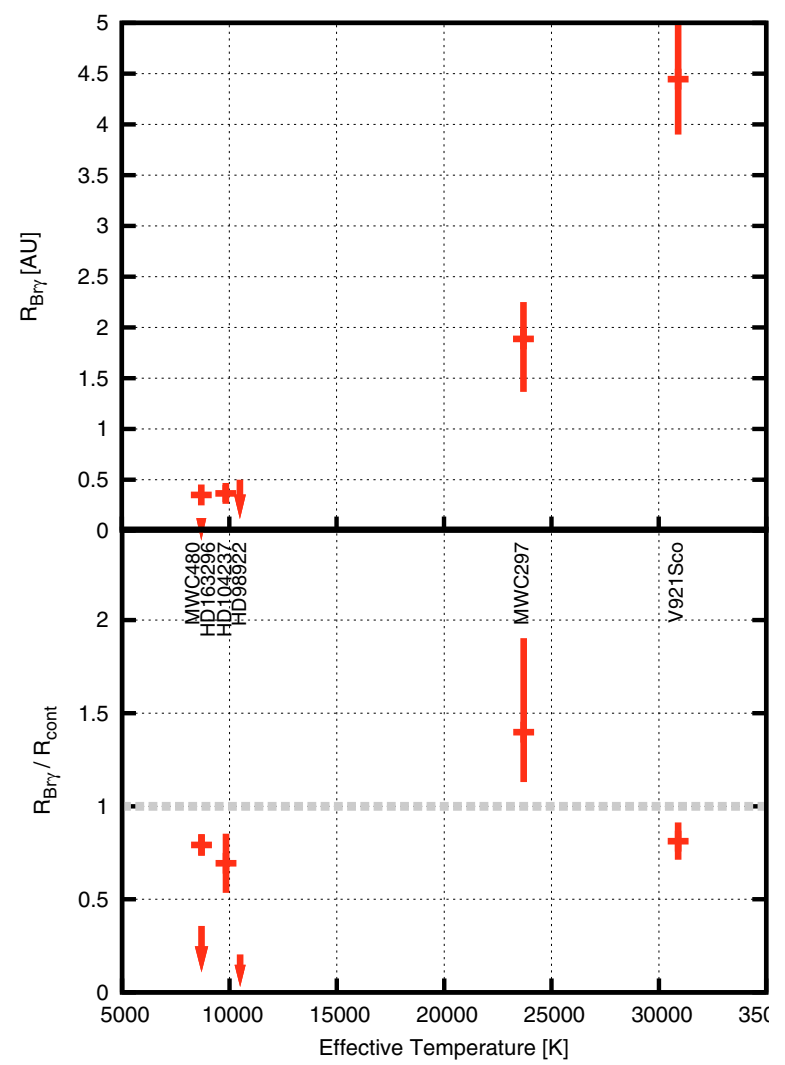

Fig. 9. $R_{\mathrm{Br} \gamma}$ and $R_{\mathrm{Br} \gamma} / R_{\text {cont }}$ plotted as a function of effective stellar temperature.

in wind models, although the influence of inclination effects cannot be ruled out at the current time.

Another spectroscopic parameter which was used extensively in the past to characterize the accretion and outflow processes around YSOs is the line profile of the Balmer lines, especially $\mathrm{H} \alpha$. Acke et al. (2005) classified the $\mathrm{H} \alpha$ line profile of all stars in our sample using the scheme "double-peaked", "singlepeaked", "P-Cygni", and "inverse P-Cygni". The six stars in the considered sample exhibit three of these four $\mathrm{H} \alpha$ line profiles (see Table 1), with the inverse P-Cygni profile being the exception $^{2}$. As discussed in Sect. 1, interpreting these line profiles without spatial information can lead to highly ambiguous results (for instance, Cidale \& Ringuelet 1993, could already reproduce three of these four line profiles by varying the velocity law for an expanding wind). Thus, it seems promising to compare the spatially resolved information about the size of the $\mathrm{Br} \gamma$-emitting region with the shape of the $\mathrm{H} \alpha$ line profile, potentially revealing correlations between the spatial distribution and the kinematics of the emitting hydrogen. In this respect, it is important to notice that even under the assumption that $\mathrm{Br} \gamma$ and $\mathrm{H} \alpha$ trace the same physical process, these lines will form in different spatial regions due to their different energy levels - with $\mathrm{Br} \gamma$ tracing higher gas densities located closer to the star.

As shown in Fig. 11, we find indications that $R_{\mathrm{Br} \gamma} / R_{\mathrm{cont}}$ depends on the $\mathrm{H} \alpha$ line profile classification:

P-Cygni: for the HAeBe stars with a P-Cygni $\mathrm{H} \alpha$-line profile (MWC 480, HD 98922), the Br $\gamma$-emitting region is very compact

2 As discussed, for example, by Walker (1972) and Sorelli et al. (1996), the inverse P-Cygni profile is generally believed to trace mass infall, but is found towards only $\sim 5 \%$ of all HAeBe stars (Acke et al. 2005) 


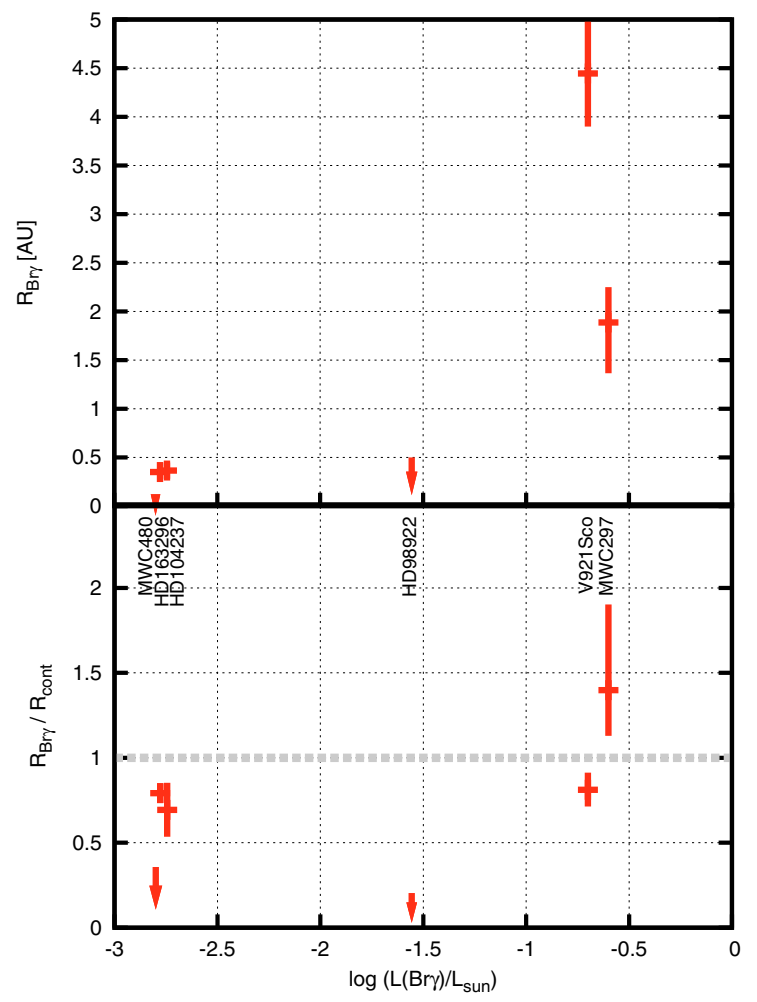

Fig. 10. $R_{\mathrm{Br} \gamma}$ and $R_{\mathrm{Br} \gamma} / R_{\text {cont }}$ plotted as a function of the $\mathrm{Br} \gamma$ line luminosity.

$\left(R_{\mathrm{Br} \gamma} / R_{\text {cont }} \lesssim 0.2\right.$, unresolved by the interferometer), consistent with an origin in a compact stellar wind, an X-wind or in magnetospheric accretion. P-Cygni $\mathrm{H} \alpha$ line profiles are generally attributed to mass outflow (Finkenzeller \& Mundt 1984; Catala \& Kunasz 1987), although for T Tauri stars Muzerolle et al. (2001) showed that these profiles can also be reproduced in magnetospheric accretion models assuming blue-shifted absorption by gas in an accretion-powered wind located outside of the magnetosphere. Therefore, the Br $\gamma$ line in MWC 480 and HD 98922 might primarily trace magnetospheric accretion, while the optical Balmer lines are strongly affected by absorption from outflowing gas. This interpretation is also consistent with the rather high accretion rates, which have been reported for these two objects (MWC 480: $\log \dot{M}_{\text {acc }} \geq-7.0 M_{\odot} \mathrm{yr}^{-1}$, Eisner 2007; HD 98922: $\log \dot{M}_{\mathrm{acc}}=-5.76 M_{\odot} \mathrm{yr}^{-1}$, Garcia Lopez et al. 2006).

Double-peaked: several theoretical studies (e.g. Cidale \& Ringuelet 1993) associate this $\mathrm{H} \alpha$ line profile with rotating gas flows, including disk winds. HD 163296, HD 104237 , and V921 Sco, which fit into this classification, exhibit a $\mathrm{Br} \gamma$-emitting region which is only slightly more compact than the dust-sublimation radius (but still spatially resolved by our spectro-interferometric measurements). Considering the gaseous inner disk scenario, Muzerolle et al. (2004) modeled the continuum and line emission from such a disk and found that for low mass accretion rates $\left(\log \dot{M}_{\text {acc }}<-7 M_{\odot} \mathrm{yr}^{-1}\right)$, the flux contribution from the gaseous inner disk is negligible. This leads us to favor the stellar wind or disk wind scenario for these three objects.

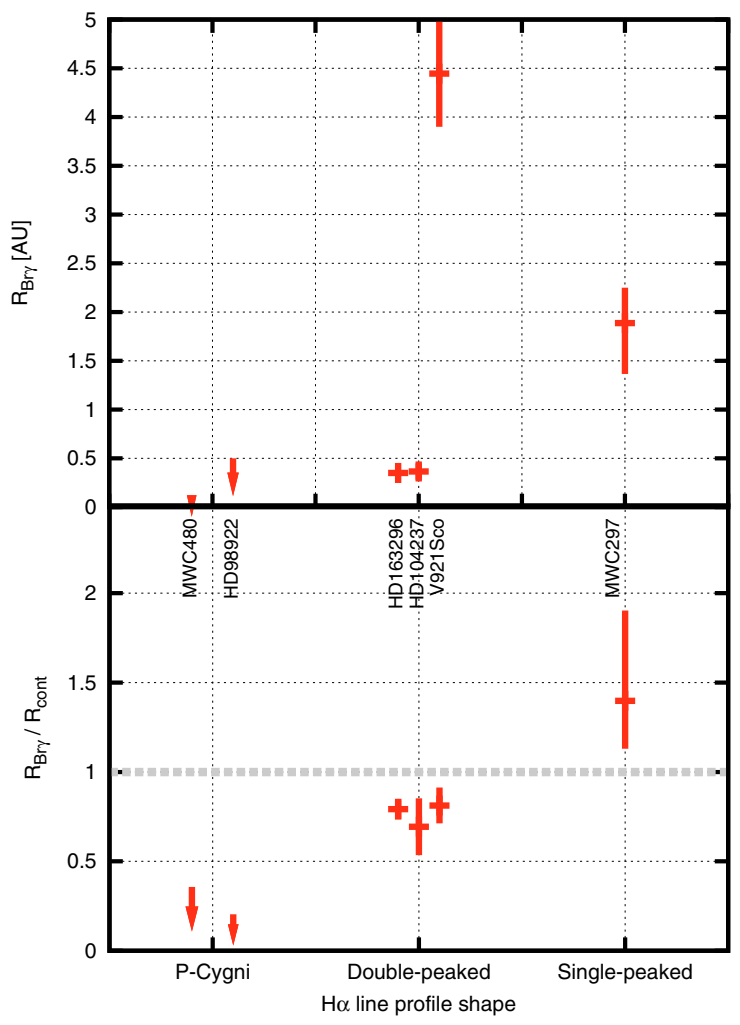

Fig. 11. $R_{\mathrm{Br} \gamma}$ and $R_{\mathrm{Br} \gamma} / R_{\text {cont }}$ versus the $\mathrm{H} \alpha$ line profile (see Table 1).

Single-peaked: exhibiting a single-peaked $\mathrm{H} \alpha$-line profile, MWC 297 shows a rather extended Br $\gamma$-emitting region, exceeding even the size of the continuum-emitting region. However, comparing the size of the $\mathrm{Br} \gamma$-emitting region around MWC 297 to sources with double-peaked $\mathrm{H} \alpha$-line profiles (such as V921 Sco), we do not find fundamental differences within the measurement uncertainties (especially taking the indications for a particularly compact continuum-emitting region around MWC 297 into account, as discussed in Sect. 6.4). Theoretical models have associated single-peaked $\mathrm{H} \alpha$ line profiles with stellar winds or with disk winds (Cidale \& Ringuelet 1993), especially for massive YSOs (Sim et al. 2005).

Based on the currently available spectroscopic and longbaseline spectro-interferometric data, it seems not feasible to discern between stellar wind and disk wind scenarios. This is due to the fact that the gas velocity field in stellar winds as well as in disk winds, comprise both rotating and expanding velocity components, which can result in similar line profiles as well as in similar spatial scales for the line-emitting region. To solve these ambiguities, future observations will require a very good sampling of the line visibility function in order to measure the subtle imprints resulting from the differences of the gas kinematics close to the star.

\subsubsection{Implications on the empirical $L(\operatorname{Br} \gamma)-L_{\text {acc }}$ relation and the accretion-outflow connection}

Within the last decade, a correlation between the Bry line luminosity and the mass accretion rate (as independently determined from the UV excess, which is commonly attributed to the accretion shock) could be established for pre-main-sequence stars with masses between $\sim 0.01$ (Natta et al. 2004) and $\sim 4 M_{\odot}$ (Muzerolle et al. 1998b; van den Ancker 2005). Being applicable even in regions of high extinction and for very low mass 
accretion rates, this correlation is of considerable practical importance, although neither the traced processes nor the underlying physical mechanisms are yet identified. It was suggested that the $\operatorname{Br} \gamma$ line emission might be a direct tracer of mass accretion, and that for large accretion rates $\left(\dot{M}_{\mathrm{acc}}>\right.$ $\left.10^{-7} M_{\odot} \mathrm{yr}^{-1}\right)$ the contributions from other processes might be negligible (van den Ancker 2005). However, attempts to reproduce the $L(\mathrm{Br} \gamma)-L_{\mathrm{acc}}$ relation using magnetospheric accretion models were not successful, but found instead that the $\operatorname{Br} \gamma$ line luminosity depends mainly on other parameters such as the size of the magnetosphere and the gas temperatures (Muzerolle et al. 1998a,b).

Based on our spatially resolved observations of the $\mathrm{Br} \gamma$-emitting region around five Herbig Ae/Be stars, we cannot support the scenario that $\mathrm{Br} \gamma$ generally traces gas located in the magnetospheric infall zone. Considering only those stars in our sample which are within the current calibration range of the relation (i.e. rejecting the early Herbig Be stars), we find that for one star (HD 98922) the size of the Br $\gamma$-emitting region is consistent with magnetospheric accretion, while two stars (HD 104237, HD 163296) exhibit a Br $\gamma$-emitting region which is more consistent with an extended stellar wind or a disk wind scenario $\left(R_{\mathrm{Br} \gamma} \gtrsim 0.6 R_{\text {cont }}\right)$. Therefore, our observations imply that, at least for some $\mathrm{HAeBe}$ stars, $\mathrm{Br} \gamma$ is not a primary tracer of accretion, but likely indirectly linked to the accretion rate, e.g. via accretion-driven mass loss. This ambivalent origin of the line emission (tracing partially infalling and outflowing matter) makes the empirical correlation between $L(\mathrm{Br} \gamma)$ and $L_{\mathrm{acc}}$ even more remarkable and suggests a tight, quantitative connection between the accretion and ejection processes in YSOs. The existence of such a connection has already been suggested based on measurements of the luminosity of accretion and outflow-tracer lines, yielding an ejection efficiency of $\dot{M}_{\text {eject }} / \dot{M}_{\text {acc }} \approx 0.1-0.2$ (Cabrit et al. 1990; Hartigan et al. 1994).

\subsubsection{Implications on the jet-launching mechanism for Herbig Ae/Be stars}

Our measurement of rather extended $\mathrm{Br} \gamma$-emitting regions also has important implications on the launching mechanism for HAeBe jets. Although most stars in our sample show only indirect signatures of outflow activity (e.g. CO outflows, outflowtracing line emission), HD 104237 and HD 163296 are also known to be associated with collimated microjets. On large scales, the interaction region between the jet ejecta and ambient material is traced by Herbig-Haro objects, while on arcsecondscales, the jets were also imaged with the HST in forbidden lines and in the hydrogen H $\alpha$ and Ly $\alpha$ transitions (Grady et al. 2004; Wassell et al. 2006).

Therefore, it seems likely that our AMBER Br $\gamma$ observations trace ionized gas located at the base of these microjets, just experiencing the acceleration and magnetic confinement processes which are required to achieve the highly collimated gas flows detected by HST. Since it is expected that stellar winds, lacking any collimation mechanism, result in wide-angle outflows, we clearly favor the disk wind model for these objects in order to explain the observational evidence for $(a)$ a rather extended Br $\gamma$-emitting region on sub-AU scales and, simultaneously; (b) collimated narrow-angle microjets on scales of several hundred AU. This main conclusion is in qualitative agreement with HST spectroscopic observations, which measured the jet opening angle and the toroidal velocity field on spatial scales $\sim 50$ AU from the base. Traced back to the footpoint of the jet, the measured velocity fields seem more consistent with a launching of the jet from the disk and not an X-wind or stellar wind (Bacciotti et al. 2002; Testi et al. 2002; Coffey et al. 2007).

Future spectro-interferometric observations will allow us to confirm this hypothesis by measuring the spatial distribution and kinematics of the line-emitting gas and comparing it with the orientation and velocity of the large-scale outflow structure.

\section{Conclusions}

We summarize the results from our investigation using VLTI/AMBER data of five HAeBe stars as follows:

1 . The $K$-band continuum-emitting region was spatially resolved for all objects. For the Herbig Ae and late Herbig Be stars the characteristic size of this region scales roughly with the square-root of the stellar luminosity, supporting the scenario that for these sources, the continuum is dominated by thermal emission from the inner rim of the dust disk. For the early-type Herbig Be stars MWC 297 and V921 Sco, on the other hand, the continuum-emitting region is significantly more compact than predicted by the $R_{\text {subl }} \propto L_{\star}^{1 / 2}$ relation, maybe suggesting that for these objects the NIR continuum is dominated by hot gas emission. Fits of inclined ring geometries to the visibilities measured on HD 163296 indicate object elongation, consistent with a notably inclined disk geometry, while for HD 98922 and V921 Sco we find no indications for a large disk inclination.

2. Closure phases were measured for four objects in the $K$-band continuum regime. For V921 Sco we measured the $\mathrm{CP}$ also within the spectrally resolved $\mathrm{Br} \gamma$ line, suggesting a nearly centro-symmetric line-emitting region (in agreement with the low inclination angle).

3. In the spectrally resolved $\operatorname{Br} \gamma$ lines of HD 163296, HD 104237, HD 98922, and V921 Sco, we measure an increase of visibility, indicating that the line-emitting region is more compact than the continuum-emitting region. MWC 297 shows a decrease of visibility within the $\mathrm{Br} \gamma$-line, although this is likely related to the compactness of the continuum-emitting region and not to fundamental differences in the line formation mechanism.

4. Judging on the currently available limited statistics, we do not find a trend which relates the size of the $\operatorname{Br} \gamma$ lineemitting region with the stellar luminosity, as was proposed by Eisner (2007). Maybe the most remarkable correlation we found was that stars with a P-Cygni $\mathrm{H} \alpha$ line profile and a high mass-accretion rate seem to show particularly compact $\mathrm{Br} \gamma$-emitting regions $\left(R_{\mathrm{Br} \gamma} / R_{\text {cont }}<0.2\right)$, while stars with a double-peaked or single-peaked $\mathrm{H} \alpha$-line profile show a significantly more extended $\mathrm{Br} \gamma$-emitting region $\left(0.6 \lesssim R_{\mathrm{Br} \gamma} / R_{\text {cont }} \lesssim 1.4\right)$.

5. Only for HD 98922, the emitting-region is compact enough to support the hypothesis that most of the $\mathrm{Br} \gamma$-emission emerges from magnetospheric accretion columns. In the other cases, the Br $\gamma$-emitting region is only slightly more compact than the dust sublimation radius, supporting the idea that the emission mainly emerges from an extended stellar wind or a disk-wind. This makes it unlikely that Bry primarily traces magnetospheric accretion in HAeBe stars, suggesting that even for Herbig Ae stars, this line is only an indirect tracer of the mass accretion rate.

Acknowledgements. We are grateful to A. Chelli and G. Duvert for carrying out independent checks on the AMBER data reduction. Furthermore, we would like to thank the referee for his comments, which helped to improve the paper. This publication makes use of data products from the Two Micron All Sky Survey, 
which is a joint project of the University of Massachusetts and the Infrared Processing and Analysis Center/California Institute of Technology, funded by the National Aeronautics and Space Administration and the National Science Foundation. NSO/Kitt Peak FTS data used here were produced by NSF/NOAO.

\section{References}

Acke, B., van den Ancker, M. E., \& Dullemond, C. P. 2005, A\&A, 436, 209 Acke, B., Verhoelst, T., van den Ancker, M. E., et al. 2008, A\&A, 485, 209 Bacciotti, F., Ray, T. P., Mundt, R., Eislöffel, J., \& Solf, J. 2002, ApJ, 576, 222 Benedettini, M., Nisini, B., Giannini, T., et al. 1998, A\&A, 339, 159 Blandford, R. D., \& Payne, D. G. 1982, MNRAS, 199, 883

Blondel, P. F. C., \& Djie, H. R. E. T. A. 2006, A\&A, 456, 1045

Böhm, T., Catala, C., Balona, L., \& Carter, B. 2004, A\&A, 427, 907

Borges Fernandes, M., Kraus, M., Lorenz Martins, S., \& de Araújo, F. X. 2007, MNRAS, 377, 1343

Brittain, S. D., Simon, T., Najita, J. R., \& Rettig, T. W. 2007, ApJ, 659, 685 Cabrit, S., Edwards, S., Strom, S. E., \& Strom, K. M. 1990, ApJ, 354, 687

Calvet, N., Muzerolle, J., Briceño, C., et al. 2004, AJ, 128, 1294

Catala, C., \& Kunasz, P. B. 1987, A\&A, 174, 158

Catala, C., Donati, J. F., Böhm, T., et al. 1999, A\&A, 345, 884

Causi, G. Li, Antoniucci, S., \& Tatulli, E. 2008, A\&A, 479, 589

Cidale, L. S., \& Ringuelet, A. E. 1993, ApJ, 411, 874

Coffey, D., Bacciotti, F., Ray, T. P., Eislöffel, J., \& Woitas, J. 2007, ApJ, 663, 350

Corcoran, M., \& Ray, T. P. 1998, A\&A, 331, 147

de Winter, D., \& The, P. S. 1990, Ap\&SS, 166, 99

Donati, J.-F., Semel, M., Carter, B. D., Rees, D. E., \& Collier Cameron, A. 1997, MNRAS, 291, 658

Drew, J. E., Busfield, G., Hoare, M. G., et al. 1997, MNRAS, 286, 538

Dullemond, C. P., Dominik, C., \& Natta, A. 2001, ApJ, 560, 957

Eisner, J. A. 2007, Nature, 447, 562

Eisner, J. A., Lane, B. F., Hillenbrand, L. A., Akeson, R. L., \& Sargent, A. I. 2004, ApJ, 613, 1049

Ferreira, J. 1997, A\&A, 319, 340

Ferreira, J., Dougados, C., \& Cabrit, S. 2006, A\&A, 453, 785

Finkenzeller, U. 1985, A\&A, 151, 340

Finkenzeller, U., \& Mundt, R. 1984, A\&AS, 55, 109

Garcia Lopez, R., Natta, A., Testi, L., \& Habart, E. 2006, A\&A, 459, 837

Grady, C. A., Perez, M. R., Talavera, A., et al. 1996, A\&AS, 120, 157

Grady, C. A., Devine, D., Woodgate, B., et al. 2000, ApJ, 544, 895

Grady, C. A., Woodgate, B., Torres, C. A. O., et al. 2004, ApJ, 608, 809

Habart, E., Testi, L., Natta, A., \& Vanzi, L. 2003, A\&A, 400, 575

Hartigan, P., Morse, J. A., \& Raymond, J. 1994, ApJ, 436, 125

Henning, T., Burkert, A., Launhardt, R., Leinert, C., \& Stecklum, B. 1998, A\&A, 336,565

Higdon, S. J. U., Devost, D., Higdon, J. L., et al. 2004, PASP, 116, 975

Hutsemekers, D., \& van Drom, E. 1990, A\&A, 238, 134

Isella, A., \& Natta, A. 2005, A\&A, 438, 899

Isella, A., Testi, L., Natta, A., et al. 2007, A\&A, 469, 213
Kraus, S., Preibisch, T., \& Ohnaka, K. 2008, ApJ, 676, 490

Leinert, C., van Boekel, R., Waters, L. B. F. M., et al. 2004, A\&A, 423, 537

Malbet, F., Benisty, M., de Wit, W.-J., et al. 2007, A\&A, 464, 43

Malfait, K., Bogaert, E., \& Waelkens, C. 1998, A\&A, 331, 211

Mannings, V., \& Sargent, A. I. 1997, ApJ, 490, 792

Mathis, J. S. 1990, ARA\&A, 28, 37

Mestel, L. 1968, MNRAS, 138, 359

Millan-Gabet, R., Schloerb, F. P., \& Traub, W. A. 2001, ApJ, 546, 358

Monnier, J. D., \& Millan-Gabet, R. 2002, ApJ, 579, 694

Monnier, J. D., Millan-Gabet, R., Billmeier, R., et al. 2005, ApJ, 624, 832

Monnier, J. D., Berger, J.-P., Millan-Gabet, R., et al. 2006, ApJ, 647, 444

Muzerolle, J., Calvet, N., \& Hartmann, L. 1998a, ApJ, 492, 743

Muzerolle, J., Hartmann, L., \& Calvet, N. 1998b, AJ, 116, 2965

Muzerolle, J., Calvet, N., \& Hartmann, L. 2001, ApJ, 550, 944

Muzerolle, J., D’Alessio, P., Calvet, N., \& Hartmann, L. 2004, ApJ, 617, 406

Natta, A., Palla, F., Butner, H. M., Evans, II, N. J., \& Harvey, P. M. 1993, ApJ, 406,674

Natta, A., Prusti, T., Neri, R., et al. 2001, A\&A, 371, 186

Natta, A., Testi, L., Muzerolle, J., et al. 2004, A\&A, 424, 603

Nisini, B., Milillo, A., Saraceno, P., \& Vitali, F. 1995, A\&A, 302, 169

Oudmaijer, R. D., \& Drew, J. E. 1999, MNRAS, 305, 166

Petrov, R. G., Malbet, F., Weigelt, G., et al. 2007, A\&A, 464, 1

Pogodin, M. A. 1994, A\&A, 282, 141

Pudritz, R. E., \& Norman, C. A. 1983, ApJ, 274, 677

Richichi, A., Percheron, I., \& Khristoforova, M. 2005, A\&A, 431, 773

Shu, F., Najita, J., Ostriker, E., et al. 1994, ApJ, 429, 781

Sim, S. A., Drew, J. E., \& Long, K. S. 2005, MNRAS, 363, 615

Sorelli, C., Grinin, V. P., \& Natta, A. 1996, A\&A, 309, 155

Stee, P., \& Bittar, J. 2001, A\&A, 367, 532

Strafella, F., Pezzuto, S., Corciulo, G. G., Bianchini, A., \& Vittone, A. A. 1998, ApJ, 505, 299

Tannirkulam, A., Monnier, J. D., Millan-Gabet, R., et al. 2008, ApJ, 677, L51

Tatulli, E., Isella, A., Natta, A., et al. 2007a, A\&A, 464, 55

Tatulli, E., Millour, F., Chelli, A., et al. 2007b, A\&A, 464, 29

Testi, L., Bacciotti, F., Sargent, A. I., Ray, T. P., \& Eislöffel, J. 2002, A\&A, 394, L31

van Boekel, R., Waters, L. B. F. M., Dominik, C., et al. 2003, A\&A, 400, L21

van den Ancker, M. E. 2005, in High Resolution Infrared Spectroscopy in

Astronomy, ed. H. U. Käufl, R. Siebenmorgen, \& A. F. M. Moorwood, 309

van den Ancker, M. E., de Winter, D., \& Tjin A Djie, H. R. E. 1998, A\&A, 330, 145

van den Ancker, M. E., Bouwman, J., Wesselius, P. R., et al. 2000, A\&A, 357, 325

van den Bergh, S., \& Herbst, W. 1975, AJ, 80, 208

Vinković, D., \& Jurkić, T. 2007, ApJ, 658, 462

Walker, M. F. 1972, ApJ, 175, 89

Wang, S., \& Looney, L. W. 2007, ApJ, 659, 1360

Wassell, E. J., Grady, C. A., Woodgate, B., Kimble, R. A., \& Bruhweiler, F. C. 2006, ApJ, 650, 985

Weigelt, G., Kraus, S., Driebe, T., et al. 2007, A\&A, 464, 87 